M. Itô

Nagoya Math. J.

Vol. 75 (1979), 1-39

\title{
SUR LES NOYAUX DE CONVOLUTION CONDITIONNELLEMENT SOUS-MÉDIANS II
}

\author{
MASAYUKI ITÔ
}

\section{§1. Introduction}

Dans toute la suite $X$ désignera un groupe abélien localement compact, séparé et dénombrable à l'infini et $\xi$ désignera la mesure de Haar sur X. Un noyau de convolution $N$ sur $X$ est une mesure de Radon positive dans $X$ et, pour une mesure de Radon réelle $\mu$ dans $X$, le $N$ potentiel de $\mu$ est la convolution $N * \mu$ dès qu'elle a un sens.

Depuis que O. Frostman [5] a étudié le principe classique du maximum pour les noyaux de Riesz-Frostman sur l'espace euclidien $\boldsymbol{R}^{n}(n \geqq 1)$, il y avait beaucoup de travaux sur le principe classique du maximum.

Dans le cas où $X$ est un tore, il y a un travail important concernant le principe classique du maximum pour les noyaux de convolution symétriques (voir [12]): Un noyau de convolution symétrique $N$ vérifie le principe classique du maximum si et seulement si la transformée de Fourier de $N$ est de la forme $\hat{N}=\lambda_{1} / \lambda_{2}$, où $\lambda_{j}(j=1,2)$ est une fonction définie-négative sur le groupe dual $\hat{X}$ de $X$ à valeurs non-négatives.

Dans le cas où $X$ est non-compact et où il n'existe aucun sousgroupe compact de $X$ excepté $\{0\}$, nous avons donné une caractérisation analogue pour les noyaux de convolution symétriques et s'annulant à l'infini (voir [7]). On se trouve quelques démonstration incomplètes dans [7].

Remarquons que si, pour un noyau de convolution $N, \hat{N}$ est, pour deux fonctions définie-négatives $\lambda_{1}$ et $\lambda_{2}$ sur $\hat{X}$, de la forme $\hat{N}=\lambda_{1} / \lambda_{2}$, alors $N$ est conditionnellement sous-médian par rapport au semi-groupe vaguement continu $\left(\alpha_{t}\right)_{t \geqq 0}$ défini par $\hat{\alpha}_{t}=\exp \left(-t \lambda_{2}\right)$.

On dit qu'un noyau de convolution $N$ est conditionnellement sousmédian par rapport au semi-groupe vaguement continu $\left(\alpha_{t}\right)_{t \geqq 0}$ donné si,

Received September 1, 1977. 
pour $t>0$ quelconque, $N * \alpha_{t}$ a un sens, si, en posant $\mathscr{D}^{+}\left(\lim _{t \rightarrow 0} \frac{1}{t}\left(N * \alpha_{t}-N\right)\right)$ l'ensemble des fonctions $f \in C_{K}^{+}(X)$ vérifiant la condition que $\frac{1}{t}\left(N * \alpha_{t}-N\right) * f$ converge uniformément sur tout compact lorsque $t \rightarrow 0, \mathscr{D}^{+}\left(\lim _{t \rightarrow 0} \frac{1}{t}\left(N * \alpha_{t}-N\right)\right)$ est dense dans $C_{K}^{+}(X)$ et si, pour $f \in \mathscr{D}^{+}\left(\lim _{t \rightarrow 0} \frac{1}{t}\left(N * \alpha_{t}-N\right)\right)$ à support $\subset C\{0\}$ quelconque, $\lim _{t \rightarrow 0} \frac{1}{t}\left(N * \alpha_{t}-N\right) * f(0) \geqq 0$. Dans ce cas, il existe une mesure de Radon positive $\sigma$ en dehors de l'origine, et une seule telle que, pour $f \in \mathscr{D}^{+}\left(\lim _{t \rightarrow 0} \frac{1}{t}\left(N * \alpha_{t}-N\right)\right)$ à support $\subset C\{0\}$ quelconque, $\int f d \sigma=\lim _{t \rightarrow 0} \frac{1}{t}\left(N * \alpha_{t}\right.$ $-N) * f(0)$.

Supposons qu'il n'existe aucun sous-groupe compact excepté $\{0\}$. Le but de cet article est de montrer l'équivalence entre les deux énoncés suivants (a) et (b). Soient $N$ un noyau de convolution et $N^{\prime}$ un noyau de convolution vérifiant $N=o\left(N^{\prime}\right)$. On désigne par $\varepsilon$ la mesure d'unité à l'origine.

(a) $N$ vérifie le principe de domination relatif à $N^{\prime}$.

(b) Il existe un semi-groupe vaguement continu $\left(\alpha_{t}\right)_{t \geqq 0}$ des mesures de Radon positives vérifiant $\alpha_{t} \neq \varepsilon(t>0)$ tel que $N$ soit conditionnellement sous-médian par rapport à $\left(\alpha_{t}\right)_{t \geqq 0}$ et que, pour $t \geqq 0$ quelconque, $N^{\prime} \geqq N^{\prime} * \alpha_{t}$ dans $X$.

Ceci donnera immédiatement l'équivalence suivante:

Pour qu'un noyau de convolution $N$ s'annulant à l'infini vérifie le principe classique du maximum, il faut et il suffit qu'il existe un semigroupe vaguement continu et sous-markovien $\left(\alpha_{t}\right)_{t \geqq 0}$ des mesures de Radon positives vérifiant $\alpha_{t} \neq \varepsilon(t>0)$ tel que $N$ soit conditionnellement sousmédian par rapport à $\left(\alpha_{t}\right)_{t \geqq 0}$.

Dans le cas où $X=R^{n}, N$ vérifie le principe classique du maximum si et seulement s'il existe un laplacien généralisé $L \neq 0$ tel que $L * N \geqq 0$ au sens des distributions en dehors de l'origine.

\section{§2. Préliminaires et quelques propositions}

Dans ce paragraphe, on ne supposera aucune condition supplémentaire pour $X$.

On désigne par $C_{K}=C_{K}(X)$ l'espace vectoriel topologique usuel des 
fonctions finies et continues dans $X$ à support compact et par $M_{K}=M_{K}(X)$ l'ensemble formé par toutes les fonctions $\xi$-mesurables et bornées dans $X$ à valeurs réelles et à support compact. Leurs sous-ensembles des fonctions $\geqq 0$ sont notés $C_{K}^{+}$et $M_{K}^{+}$, respectivement. Pour un noyau de convolution $N$ et pour $f \in M_{K}, N *(f \xi)$ est absolument continu par rapport à $\xi$, et sa densité s'écrit $N f$. Evidemment $N f$ est localement bornée.

Soient $N_{1}$ et $N_{2}$ deux noyaux de convolution. On note $N_{1}=O\left(N_{2}\right)$ si, pour $f \in C_{K}^{+}$quelconque, il existe $g \in C_{K}^{+}$et un compact $K$ dans $X$ tel que $N_{1} * f \leqq N_{2} * g$ sur $C K$. On note $N_{1}=o\left(N_{2}\right)$ si $N_{1}$ et $N_{2} \neq 0$ sont à support compact ou bien si supp $\left(N_{2}\right)$ est non-compact et si, pour $f \in C_{K}^{+}$ quelconque, il existe $g \in C_{K}^{+}$telle que $N_{2} * g(x)>0$ sur $\operatorname{supp}\left(N_{1} * f\right)$ et que

$$
\frac{N_{1} * f(x)}{N_{2} * g(x)}=o(1)
$$

dans $\left\{x \in X ; N_{2} * g(X)>0\right\}$ à l'infini.

On dit que $N_{1}$ vérifie le principe de domination relatif à (resp. le principe transitif de domination par rapport à) $N_{2}$ si, pour $f, g \in C_{K}^{+}$ quelconques, $N_{1} * f \leqq N_{2} * g$ (resp. $N_{2} * f \leqq N_{2} * g$ ) sur $X$ dès que $N_{1} * f \leqq N_{2} * g$ (resp. $N_{1} * f \leqq N_{1} * g$ ) sur le support de $f$, $\operatorname{supp}(f)$. Dans ce cas, on écrit $N_{1} \prec N_{2}$ (resp. $N_{1} \sqsubset N_{2}$ ).

Remarque 1. Supposons que $N_{1} \prec N_{2}$. Alors on a:

(2.2) Si $N_{2} \neq 0$, alors $N_{1}=O\left(N_{2}\right)$.

(2.3) Si $N_{1} \neq 0$, alors $\operatorname{supp}\left(N_{1}\right) \ni 0$.

(2.4) Si $\operatorname{supp}\left(N_{2}\right) \ni 0$, alors $\operatorname{supp}\left(N_{1}\right) \subset \operatorname{supp}\left(N_{2}\right)$.

On voit facilement (2.2) et (2.3). Pour $f \in C_{K}^{+}$et $g \in C_{K}^{+}$vérifiant $\operatorname{supp}^{\prime}(g) \subset\{x \in X ; f(x)>0\}$ quelconques, $\operatorname{supp}\left(N_{2}\right) \ni 0$ et $N_{1} \prec N_{2}$ montrent qu'il existe une constante $a>0$ vérifiant $a N_{1} * g \leqq N_{2} * f$ sur $X$, et donc $\operatorname{supp}\left(N_{1}\right)+\operatorname{supp}(f) \subset \operatorname{supp}\left(N_{2}\right)+\operatorname{supp}(f)$, où, pour deux sous-ensembles $A$ et $B$ de $X, A+B$ désigne $\{x+y ; x \in A, y \in B\}$. En faisant $\operatorname{supp}(f) \downarrow\{0\}$, on arrive à supp $\left(N_{1}\right) \subset \operatorname{supp}\left(N_{2}\right)$, d'où (2.4).

On dit que $N_{1}$ vérifie le principe du balayage relatif à $N_{2}$ (resp. le principe transitif du balayage par rapport à $N_{2}$ ) si, pour une mesure de Radon positive $\mu$ à support compact et un ouvert relativement compact $\omega$ dans $X$ quelconques, il existe une mesure de Radon positive $\mu^{\prime}$ (resp. $\mu^{\prime \prime}$ ) portée par $\bar{\omega}$ telle que: 
(2.5) $N_{1} * \mu^{\prime} \leqq N_{2} * \mu$ (resp. $\left.N_{2} * \mu^{\prime \prime} \leqq N_{2} * \mu\right)$ dans $X$.

(2.6) $N_{1} * \mu^{\prime}=N_{2} * \mu$ (resp. $N_{1} * \mu^{\prime \prime} \geqq N_{1} * \mu$ ) dans $\omega$.

Dans ce cas, on écrit $N_{1} \prec_{B} N_{2}$ (resp. $N_{1} \sqsubset_{B} N_{2}$ ), et l'on dit que $\mu^{\prime}$ (resp. $\mu^{\prime \prime}$ ) est une mesure balayée (resp. une mesure balayée transitivement) de $\mu$ sur $\omega$ relativement à $\left(N_{1}, N_{2}\right)$.

Proposition 2. Pour deux noyaux de convolution $N_{1} \neq 0$ et $N_{2} \neq 0$, les six énoncés suivants sont équivalents:

(a) $N_{1} \prec N_{2}$.

(b) $N_{1} \sqsubset N_{2}$.

(c) $N_{1} \prec_{B} N_{2}$.

(d) $N_{1} \sqsubset{ }_{B} N_{2}$.

(e) $\check{N}_{1} \prec \check{N}_{2}$

(f) Pour $c \in(0, \infty)$ quelconque, $N_{1}+c \varepsilon \prec N_{2}$.

Pour un noyau de convolution $N$, on note $\check{N}$ le noyau de convolution défini par $\int f d \check{N}=\int \check{f} d N$ pour toute $f \in C_{K}$, où $\breve{f}(x)=f(-x)$, et l'on l'appelle le noyau adjoint de $N$. On dit souvent que $\varepsilon$ est le noyau d'unité. Pour $(a) \Leftrightarrow(b) \Leftrightarrow(c) \Leftrightarrow(d)$, voir le théorème 20 dans [11]. Pour (a) $\Leftrightarrow$ (e) $\Leftrightarrow$ (f), voir les propositions 2,3 dans [10] et la proposition 4 dans [11].

Pour un noyau de convolution $N$, on désigne par $D^{+}(N)$ l'ensemble des mesures de Radon positives dont les $N$-potentiels sont définis.

Proposition 3. Soient $N$ un noyau de convolution et $N^{\prime} \neq 0$ un noyau de convolution vérifiant $N \prec N^{\prime}$. Soit $\mu$ une mesure de Radon positive appartenant à $D^{+}\left(N^{\prime}\right)$. Si $N^{\prime} \geqq N^{\prime} * \mu$ dans $X$, alors $\mu \in D^{+}(N)$ et $N(\{0\})$ $\geqq N * \mu(\{0\})$.

Démonstration. Comme $N=O\left(N^{\prime}\right)$, on a $\mu \in D^{+}(N)$. Soit $\varphi \in C_{K}^{+}$ vérifiant $\check{N}^{\prime} * \varphi(0)>0$. Pour $\delta \in(0, \infty)$ quelconque, il existe $v \in \mathscr{V}$ tel que, pour $f \in C_{K}^{+}$portée par $v$ et vérifiant $0 \leqq f \leqq 1, f(0)=1$ quelconque,

$$
\check{N} * f(x) \leqq \frac{N(\{0\})}{\check{N}^{\prime} * \varphi(0)} \check{N}^{\prime} * \varphi(x)+\delta \check{N}^{\prime} * \varphi(x) \quad \text { sur } v .
$$

On désigne ici par $\mathscr{V}$ la totalité des voisinages compacts de l'origine. Comme $\check{N} \prec \check{N^{\prime}}$, l'inégalité dans (2.7) a lieu sur $X$. En faisant supp $(f) \downarrow\{0\}$ et $\delta \downarrow 0$, on arrive à

$$
\check{N}(\{x\}) \leqq \frac{N(\{0\})}{\check{N}^{\prime} * \varphi(0)} \check{N}^{\prime} * \varphi(x) \quad \text { sur } X .
$$

On a donc 


$$
\begin{aligned}
N(\{0\})-N * \mu(\{0\}) & =N(\{0\})-\int \check{N}(\{x\}) d \mu(x) \\
& \geqq \frac{N(\{0\})}{\check{N}^{\prime} * \varphi(0)}\left(\check{N}^{\prime} * \varphi(0)-\int \check{N}^{\prime} * \varphi d \mu\right) \geqq 0,
\end{aligned}
$$

d'où la proposition 3 .

Pour un noyau de convolution $N$, on désigne par $S(N)$ l'ensemble des noyaux de convolution $N^{\prime}$ vérifiant $N \prec N^{\prime}$. En rappelant (2.8), on obtient le corollaire suivant:

Corollatre 4. Soit $N$ un noyau de convolution vérifiant $S(N) \neq \emptyset$ et $S(N) \neq\{0\}$. Si $N(\{0\})=0$, alors, pour $x \in X$ quelconque, $N(\{x\})=0$.

Pour deux noyaux de convolution $N_{1}$ et $N_{2}$, on dit que $N_{1}$ est régulier par rapport à $N_{2}$ si $\bigcap_{v \in \mathcal{r}} P\left(N_{1}, N_{2} ; v\right)=\{0\}$, où

$$
P\left(N_{1}, N_{2} ; v\right)=\left\{\overline{\left.N_{1} * \lambda ; \lambda \in D^{+}\left(N_{1}\right) \cap D^{+}\left(N_{2}\right), \operatorname{supp}(\lambda) \subset C v, N_{2} \geqq N_{2} * \lambda\right\}}\right.
$$

où l'adhérence est au sens de la topologie vague. On dit simplement qu'un noyau de convolution $N$ est régulier si $N$ est régulier par rapport à lui-même (cf. [11]). On écrit simplement $P(N ; v)=P(N, N ; v)$.

Remarque 5. Soient $N_{1}$ et $N_{2}$ deux noyaux de convolution. Si $N_{1}=o\left(N_{2}\right)$ ou bien si $N_{1}=O\left(N_{2}\right)$ et $N_{2}$ est régulier, alors $N_{1}$ est régulier par rapport à $N_{2}$.

En effet, soit $\eta \in \bigcap_{v \in \mathscr{r}} P\left(N_{1}, N_{2} ; v\right)$ quelconque. Alors il existe une famille filtrante à droite $\left(v_{\alpha}\right)_{\alpha \in A} \subset \mathscr{V}$ et une famille filtrante $\left(N_{1} * \lambda_{\alpha}\right)_{\alpha \in A}$ des $N_{1}$-potentiels des mesures de Radon positives telles que $\bigcup_{\alpha \in \Lambda} v_{\alpha}=X$, $\operatorname{supp}\left(\lambda_{\alpha}\right) \subset C v_{\alpha}, \lim _{\alpha} N_{1} * \lambda_{\alpha}=\eta$ (vaguement) et que $N_{2} \geqq N_{2} * \lambda_{\alpha}$. Si $N_{1}=o\left(N_{2}\right)$, on a $\lim _{\alpha} N_{1} * \lambda_{\alpha}=0$ (vaguement), d'après la remarque 30 dans [11]. Si $N_{2}$ est régulier, alors on a $\lim N_{2} * \lambda_{\alpha}=0$ (vaguement). Comme $N_{1}=O\left(N_{2}\right)$, on a aussi $\lim _{\alpha} N_{1} * \lambda_{\alpha}=0$ (vaguement). Par conséquent, $\eta=0$, d'où $\bigcap_{v \in \mathcal{r}} P\left(N_{1}, N_{2} ; v\right)=\{0\}$.

On voit aussi facilement que si $N_{1}=O\left(N_{2}\right)$ et $N_{2}$ est régulier, alors $N_{1}$ est aussi régulier.

Remarque 6. En rappelant la démonstration du théorème 20 et la proposition 25 dans [11], on voit l'énoncé suivant:

Soit $N$ un noyau de convolution vérifiant $S(N) \neq \emptyset$ et $S(N) \neq\{0\}$. 
Pour un ouvert relativement compact $\omega$ dans $X$, il existe une mesure de Radon positive $\mu_{\omega}^{\prime \prime}$ portée par $\bar{\omega}$ telle que l'on ait:

$$
N(\{0\}) \mu_{\omega}^{\prime \prime} \leqq N \text { dans } X .
$$

(2.12) Pour $N^{\prime} \in S(N)$ quelconque, $\mu_{\omega}^{\prime \prime}$ est une mesure balayée transitivement de $\varepsilon$ sur $\omega$ relativement à $\left(N, N^{\prime}\right)$.

(2.13) Pour une mesure de Radon positive $\nu$ appartenant à $D^{+}(N)$ et $N^{\prime} \in S(N)$ quelconques, $N^{\prime} * \nu \geqq N^{\prime} * \mu_{\omega}^{\prime \prime}$ dans $X$ dès que $N * \nu \geqq N * \mu$ dans $\omega$.

Dans ce cas, $\mu_{\omega}^{\prime \prime}$ s'appelle une mesure balayée transitivement de $\varepsilon$ sur $\omega$ par rapport à $S(N)$.

Remarque 7. Soit $N$ un noyau de convolution. Supposons qu'il existe un noyau de convolution $N^{\prime} \neq 0$ dans $S(N)$ telle que $N$ soit régulier par rapport à $N^{\prime}$. Alors, pour un ouvert quelconque $\omega$ dans $X$, il existe une mesure de Radon positive $\mu_{\omega}^{\prime \prime}$ portée par $\bar{\omega}$ vérifiant (2.11), (2.12) et (2.13).

En effet, on choisit une suite croissante $\left(\omega_{n}\right)_{n=1}^{\infty}$ d'ouverts relativement compacts telle que $\bigcup_{n=1}^{\infty} \omega_{n}=\omega$. Soit $\mu_{n}^{\prime \prime}$ une mesure balayée transitivement de $\varepsilon \operatorname{sur} \omega_{n}$ par rapport à $S(N)$. Comme $N^{\prime} \geqq N^{\prime} * \mu_{n}^{\prime \prime}$ et $N=O\left(N^{\prime}\right),\left(\mu_{n}^{\prime \prime}\right)_{n=1}^{\infty}$ et $\left(N * \mu_{n}^{\prime \prime}\right)_{n=1}^{\infty}$ sont vaguement bornées. Donc on peut supposer que $\left(\mu_{n}^{\prime \prime}\right)_{n=1}^{\infty}$ et $\left(N * \mu_{n}^{\prime \prime}\right)_{n=1}^{\infty}$ convergent vaguement. Posons $\mu_{\omega}^{\prime \prime}=\lim _{n \rightarrow \infty} \mu_{n}^{\prime \prime}$ et $\eta=\lim _{n \rightarrow \infty} N * \mu_{n}^{\prime \prime}$ (vaguement). Pour que $\mu_{\omega}^{\prime \prime}$ soit une mesure demandée, il suffit que $\eta=N * \mu_{\omega}^{\prime \prime}$. Pour $\varphi \in C_{K}^{+}$vérifiant $0 \leqq \varphi \leqq 1$ quelconque, $\lim _{n \rightarrow \infty} N *\left(\varphi \mu_{n}^{\prime \prime}\right)$ $=N *\left(\varphi \mu_{\omega}^{\prime \prime}\right)$ (vaguement), et donc

$$
0 \leqq \eta-N * \varepsilon_{\omega}^{\prime \prime} \leqq \lim _{n \rightarrow \infty} N *\left((1-\varphi) \mu_{n}^{\prime \prime}\right) \text { dans } X .
$$

Ceci donne que, pour $v \in \mathscr{V}$ quelconque, il existe $\eta_{v} \in P\left(N, N^{\prime} ; v\right)$ vérifiant $0 \leqq \eta-N * \mu_{\omega}^{\prime \prime} \leqq \eta_{v}$. En faisant $v \uparrow X$, on arrive à $\eta=N * \mu_{\omega}^{\prime \prime}$, d'où la remarque 7 .

On dit aussi que $\mu_{\omega}^{\prime \prime}$ est une mesure balayée transitivement de $\varepsilon$ sur $\omega$ par rapport à $S(N)$. Dans les présentes deux remarques, pour $N^{\prime} \in S(N)$, quelconque, $N^{\prime} * \mu_{\omega}^{\prime \prime}$ est uniquement déterminé.

Proposition 8. Soit $N$ un noyau de convolution vérifiant $N(\{0\})>0$. Supposons qu'il existe un noyau de convolution $N^{\prime} \neq 0$ dans $S(N)$ tel que $N$ soit régulier par rapport à $N^{\prime}$. Alors il existe une mesure de Radon. 
positive $\mu^{\prime \prime}$ dans $X$ telle que l'on ait:

$$
\begin{gathered}
\mu^{\prime \prime}(\{0\})=0 . \\
N * \mu^{\prime \prime} \geqq N \text { dans } C\{0\} . \\
N^{\prime \prime} \geqq N^{\prime \prime} * \mu^{\prime \prime} \text { dans } X \text { pour tout } N^{\prime \prime} \in S(N) .
\end{gathered}
$$

Démonstration. Soit $\left(\omega_{\alpha}\right)_{\alpha \in \Lambda}$ une famille filtrante à droite d'ouverts relativement compacts dans $X$ telle que $\bar{\omega}_{\alpha} \subset C\{0\}$ et $\bigcup_{\alpha \in \Lambda} \omega_{\alpha}=C\{0\}$. On prend une mesure balayée transitivement $\mu_{\alpha}^{\prime \prime}$ de $\varepsilon$ sur $\omega_{\alpha}$ par rapport à $S(N)$. Alors $N * \mu_{\alpha}^{\prime \prime} \geqq N$ dans $\omega_{\alpha}, N^{\prime \prime} \geqq N^{\prime \prime} * \mu_{\alpha}^{\prime \prime}$ dans $X$ pour tout $N^{\prime \prime} \in S(N)$ et

$$
N(\{0\}) \mu_{\alpha}^{\prime \prime} \leqq N-N(\{0\}) \varepsilon .
$$

Comme $N^{\prime} \neq 0$, on peut supposer que $\left(\mu_{\alpha}^{\prime \prime}\right)_{\alpha \in A}$ converge vaguement vers une mesure de Radon positive $\mu^{\prime \prime}$. De la même manière que dans la remarque 7 , on a $\lim N * \mu_{\alpha}^{\prime \prime}=N * \mu^{\prime \prime}$ (vaguement), et donc $\mu^{\prime \prime}$ vérifie (2.16) et (2.17). D'après (2.18), on a $\mu^{\prime \prime}(\{0\})=0$. La démonstration est ainsi complète.

Dans ce cas, $\mu^{\prime \prime}$ est une mesure balayée transitivement de $\varepsilon$ sur $C\{0\}$ par rapport à $S(N)$.

Une famille $\left(N_{p}\right)_{p>0}$ des noyaux de convolution est, par définition, une résolvante si, pour $p>0$ et $q>0$ quelconques,

$$
N_{p}-N_{q}=(q-p) N_{p} * N_{q} \quad \text { (Équation résolvante). }
$$

On connaît bien que, pour un noyau de convolution $N$, une résolvante $\left(N_{p}\right)_{p>0}$ vérifiant $\lim _{p \rightarrow 0} N_{p}=N$ (vaguement) est uniquement déterminée dès qu'elle existe. Dans ce cas, en posant $N_{0}=N,\left(N_{p}\right)_{p \geqq 0}$ s'appelle la résolvante associée à $N$.

D'après le lemme 35 et la remarque 33 dans [11], on voit la remarque suivante:

Remarque 9. Pour un noyau de convolution $N$, les deux énoncés suivants sont équivalents:

(a) Il existe la résolvante associée à $N$.

(b) $N$ est régulier et vérifie le principe de domination.

On dit que $N$ vérifie le principe de domination si $N \prec N$. 
Proposition 10. Soient $\left(N_{p}\right)_{p>0}$ une résolvante et $N^{\prime}$ un noyau de convolution. Alors, pour que, pour $p>0$ quelconque, $N_{p} \prec N^{\prime}$, il faut et il suffit que, pour $p>0$ quelconque, $N^{\prime} \geqq p N^{\prime} * N_{p}$.

Démonstration. Comme $N_{p}$ vérifie le principe de domination, on a $\operatorname{supp}\left(N_{p}\right)$ ə 0 ou bien $N_{p}=0$. Donc on peut supposer que $N^{\prime} \neq 0$. D'après l'équation résolvante, on $\mathrm{a}$, pour tous $p>0$ et $q>0$,

$$
N_{q}+\frac{1}{p} \varepsilon=\frac{1}{p}\left(\varepsilon+\sum_{n=1}^{\infty}\left(p N_{p+q}\right)^{* n}\right),
$$

où $(\cdot)^{* 1}=(\cdot)$ et $(\cdot)^{* n+1}=(\cdot)^{* n} *(\cdot)$. Si $\quad N^{\prime} \geqq p N^{\prime} * N_{p+q}$, alors $N_{q}+\frac{1}{p} \varepsilon \prec N^{\prime}$ (voir la proposition 2 et la remarque 10 dans [11]). Si $N_{q}+\frac{1}{p} \varepsilon \prec_{B} N^{\prime}$, alors $N^{\prime} \geqq p N^{\prime} * N_{p+q}$. D'après la proposition 2 , on voit que $N_{q}+\frac{1}{p} \varepsilon \prec N^{\prime}$ et $N^{\prime} \geqq p N^{\prime} * N_{p+q}$ sont équivalents. Ceci donne immédiatement la proposition 10 (voir aussi la proposition 2 dans [11]).

Proposition 11. Soit $\left(N_{p}\right)_{p>0}$ une résolvante et supposons qu'il existe un noyau de convolution $N^{\prime} \neq 0$ tel que, pour $p>0$ quelconque, $N^{\prime} \geqq p N^{\prime} * N_{p}$. Alors $p N_{p}$ converge vaguement vers une mesure de Radon positive $\eta$ dans $X$ lorsque $p \rightarrow 0$. Si $\eta \neq 0$, alors, pour $p>0$ quelconque, $\operatorname{supp}\left(N_{p}\right)$ $\subset \operatorname{supp}(\eta), \eta$ est portée par un sous-groupe compact $\Gamma$ de $X$ et la restreinte de $\eta$ sur $\Gamma$ est la mesure de Haar normalisée de $\Gamma$.

Démonstration. Evidemment $\left(p N_{p}\right)_{p>0}$ est vaguement bornée. Supposons qu'il existe un point vaguement adhérent $\eta \neq 0$ de $\left(p N_{p}\right)_{p>0}$ lorsque $p \rightarrow 0$. On prend une famille filtrante $\left(N_{p_{\alpha}}\right)_{\alpha \in \Lambda}$ telle que $\lim _{\alpha} p_{\alpha}=0$ et $\lim _{\alpha} p_{\alpha} N_{p_{\alpha}}$ $=\eta$ (vaguement). En utilisant le lemme de J. Deny (voir le lemme 5.2 dans [4]) et en faisant $p_{\alpha} \rightarrow 0$ dans l'équation résolvante $\left(p_{\alpha} N_{p_{\alpha}}\right) *\left(\varepsilon-\left(p_{\beta}\right.\right.$ $\left.\left.-p_{\alpha}\right) N_{p_{\beta}}\right)=p_{\alpha} N_{p_{\beta}}(\beta \in \Lambda)$, on voit que $\eta *\left(\varepsilon-p_{\beta} N_{p_{\beta}}\right)=0$. On a aussi, pour $p>0$ quelconque,

$$
\eta *\left(\varepsilon-p N_{p}\right)=0 .
$$

En faisant $p_{\beta} \rightarrow 0$, on voit que $(\eta)^{* 2}=\eta$, en utilisant encore le lemme de J. Deny. D'après le théorème 1 dans [13], $\eta$ est portée par un sous-groupe compact $\Gamma$ de $X$ et la restreinte de $\eta$ sur $\Gamma$ est la mesure de Haar normalisée sur $\Gamma$. Soit $\eta^{\prime}$ un point vaguement adhérent de $\left(p N_{p}\right)_{p>0}$ 
lorsque $p \rightarrow 0$ quelconque. D'après (2.21) et le lemme de J. Deny, on a $\eta=\eta * \eta^{\prime}$, et donc $\eta^{\prime} \neq 0$. De la même manière que ci-dessus, on a $\eta^{\prime}=\eta * \eta^{\prime}$, d'où $\eta=\eta^{\prime}$. Ceci donne que $\lim _{p \rightarrow 0} p N_{p}=\eta$ (vaguement). D'après (2.21) et la proposition 10, on a, pour tout $p>0, N_{p} \prec \eta$, et donc $\operatorname{supp}\left(N_{p}\right) \subset \operatorname{supp}(\eta) . \quad$ La démonstration est ainsi complète.

Ensuite une famille $\left(\alpha_{t}\right)_{t \geqq 0}$ des mesures de Radon positives s'appelle un semi-groupe vaguement continu si $\alpha_{0}=\varepsilon, \alpha_{t} * \alpha_{s}=\alpha_{t+s}$ pour tous $t \geqq 0$ et $s \geqq 0$ et l'application $\boldsymbol{R}^{+} \ni t \rightarrow \alpha_{t}$ est vaguement continue, où $\boldsymbol{R}^{+}$ $=\left\{t \in \boldsymbol{R}^{1} ; t \geqq 0\right\}$. Soit $N$ un noyau de convolution. Si $N$ s'écrit, pour un semi-groupe vaguement continu $\left(\alpha_{t}\right)_{t \geqq 0}$, comme $N=\int_{0}^{\infty} \alpha_{t} d t$, alors $N$ s'appelle un noyau de convolution de Hunt. Dans ce cas, $\left(\alpha_{t}\right)_{t \geqq 0}$ est uniquement déterminé et s'appelle le semi-groupe vaguement continu associé à $N$. Soit $\left(\alpha_{t}\right)_{t \geqq 0}$ un semi-groupe vaguement continu et supposons que, pour $p>0$ quelconque, $N_{p}=\int_{0}^{\infty} \exp (-p t) \alpha_{t} d t$ est défini. Alors $\left(N_{p}\right)_{p>0}$ est une résolvante.

Remarque 12. Soit $\left(\alpha_{t}\right)_{t \geqq 0}$ un semi-groupe vaguement continu. S'il existe un noyau de convolution $N^{\prime} \neq 0$ tel que, pour $t \in R^{+}$quelconque, $N^{\prime} * \alpha_{t}$ a un sens et que $N^{\prime} \geqq N^{\prime} * \alpha_{t}$, alors, pour $p>0$ quelconque $N_{p}=\int_{0}^{\infty} \exp (-p t) \alpha_{t} d t$ est défini.

Proposition 13. Soit $\left(N_{p}\right)_{p>0}$ une résolvante. Alors, pour qu'il existe un semi-groupe vaguement continu $\left(\alpha_{t}\right)_{t \geqq 0}$ tel que, pour $p>0$ quelconque, $N_{p}=\int_{0}^{\infty} \exp (-p t) \alpha_{t} d t$, il faut et il suffit qu'il existe $p_{0}>0$ tel que $N_{p_{0}}$ soit non-périodique ${ }^{(1)}$. Dans ce cas, $\left(\alpha_{t}\right)_{t \geqq 0}$ est uniquement déterminé.

Rappelons la caractérisation d'un noyau de convolution de Hunt suivante:

Lemme 14. Soit $N$ un noyau de convolution. Alors, pour que $N$ soit un noyau de convolution de Hunt, il faut et il suffit que $N$ soit nonpériodique et qu'il existe la résolvante associée à $N$.

Voir la proposition 1 dans [8].

Démonstration de la proposition 13. D'après le présent lemme, la

(1) Un noyau de convolution $N$ est non-périodique si, pour $x \neq 0 \in X$ quelconque, $N$ $\neq N * \varepsilon_{x}$, où $\varepsilon_{x}$ désigne la mesure d'unité à $x$. 
condition est nécessaire. Montrons que la condition est suffisante. En rappelant l'équation résolvante, on voit que, pour $p>0$ quelconque, $N_{p}$ est non-périodique. D'après le lemme 14, pour $p>0$ quelconque, il existe un semi-groupe vaguement continu $\left(\alpha_{p, t}\right)_{t \geqq 0}$, et un seul tel que $N_{p}$ $=\int_{0}^{\infty} \alpha_{p, t} d t$. Comme $\left(N_{p+q}\right)_{q \geqq 0}$ est la résolvante associée à $N_{p}$, on a, pour $t \in R^{+}$quelconque, $\alpha_{p+q, t}=\exp (-q t) \alpha_{p, t}$. Par conséquent, il existe un semi-groupe vaguement continu $\left(\alpha_{t}\right)_{t \geqq 0}$, et un seul tel que, pour $p \in(0, \infty)$ et $t \in \boldsymbol{R}^{+}$quelconques, $\alpha_{p, t}=\exp (-p t) \alpha_{t}$, d'où la proposition 13.

On dit que $\left(\alpha_{t}\right)_{t \geqq 0}$ est le semi-groupe vaguement continu associé à $\left(N_{p}\right)_{p>0}$

Proposition 15. Soit $\left(N_{p}\right)_{p>0}$ une résolvante des noyaux de convolution $\neq 0$ vérifiant $\lim _{p \rightarrow 0} p N_{p}=0$ (vaguement) et supposons qu'il existe un noyau de convolution $N^{\prime} \neq 0$ tel que, pour tout $p>0, N^{\prime} \geqq p N^{\prime} * N_{p}$ dans $X$. Pour $v \in \mathscr{V}$ quelconque, on désigne par $\mu_{p, c v}^{\prime}$ une mesure balayée de $\varepsilon$ sur $C v$ relativement $\grave{a} N_{p}^{(2)}$. Alors $\left(\mu_{p, C v}^{\prime}\right)_{p>0}$ est vaguement bornée et $\left(N_{p}\right.$ $\left.-N_{p} * \mu_{p, C v}^{\prime}\right)_{p>0}$ converge vaguement lorsque $p \rightarrow 0$. Posons $\eta_{v}$ sa limite vague. Alors, pour $p>0$ et un point vaguement adhérent $\mu_{C v}^{\prime}$ de $\left(\mu_{p, C v}^{\prime}\right)_{p>0}$ lorsque $p \rightarrow 0$ quelconques,

$$
\eta_{v} *\left(\varepsilon-p N_{p}\right)=N_{p} *\left(\varepsilon-\mu_{C v}^{\prime}\right) .
$$

En particulier, si, pour tout $p>0, N_{p}$ est non-périodique, alors $\eta_{v} \neq 0$ et $\left(\mu_{p, C v}^{\prime}\right)_{p>0}$ converge vaguement lorsque $p \rightarrow 0$.

Démonstration. Admettons la première partie et supposons que, pour tout $p>0, N_{p}$ est non-périodique. Alors $N_{p}$ est un noyau de convolution de Hunt (voir le lemme 14). D'après (2.22) et l'injectivité de $N_{p}{ }^{(3)}$, on voit que $\left(\mu_{p, C v}^{\prime}\right)_{p>0}$ converge vaguement lorsque $p \rightarrow 0$ et que $\eta_{v} \neq 0$.

Montrons la première partie. D'après la proposition 10, on $\mathrm{a}$, pour tout $p>0, N_{p} \prec N^{\prime}$, et donc la proposition 2 donne que $N_{p} \sqsubset N^{\prime}$. Ceci

(2) Pour une mesure de Radon positive $\mu$ à support compact et pour un ouvert $\omega$ dans $X, \mu^{\prime}$ est une mesure balayée de $\mu$ sur $\omega$ relativement à un noyau convolution $N$ si $\operatorname{supp}\left(\mu^{\prime}\right) \subset \bar{\omega}, N * \mu^{\prime} \leqq N * \mu$ dans $X, N * \mu^{\prime}=N * \mu^{\prime}$ dans $\omega$ et si, pour une mesure de Radon positive $\nu$ appatenant à $D^{+}(N)$ quelconque, $N * \nu \leqq N * \mu^{\prime}$ dans $X$ dès que $N * \nu \leqq N * \mu$ dans $\omega$. Si $N$ est un noyau de convolution de Hunt, alors $\mu^{\prime}$ est uniquement déterminée.

(3) On dit qu'un noyau de convolution $N$ est injectif si, pour $\mu, \nu$ de $D^{+}(N)$ quelconques, $\mu=\nu$ dès que $N * \mu=N * \nu$. Il est bien connu qu'un noyau de convolution de Hunt est injectif (voir, par exemple, [4]). 
donne que

$$
N^{\prime} \geqq N^{\prime} * \mu_{p, C v}^{\prime} \text { dans } X(p>0) .
$$

Par conséquent $\left(\mu_{p, C v}^{\prime}\right)_{p>0}$ est vaguement bornée. Montrons que la famille $\left(N_{p}-N_{p} * \mu_{p, C v}^{\prime}\right)_{p>0}$ est aussi vaguement bornée. Supposons qu'elle ne l'est plus. Alors il existe une suite $\left(p_{n}\right)_{n=1}^{\infty}$ des nombres positifs telle que l'on ait $\lim _{n \rightarrow \infty} p_{n}=0$ et $\lim _{n \rightarrow \infty} \int d\left(N_{p_{n}}-N_{p_{n}} * \mu_{p_{n}, C v}^{\prime}\right)=\infty$. On peut supposer ici que $\left(\mu_{p_{n}, C v}^{\prime}\right)_{n=1}^{\infty}$ converge vaguement vers une mesure de Radon positive $\mu_{C v}^{\prime}$ portée par $\overline{C v}$ lorsque $n \rightarrow \infty$. Posons $c_{n}=\int d\left(N_{p_{n}}-N_{p_{n}} * \mu_{p_{n}, C v}^{\prime}\right)$ et $\eta_{n}$ $=\frac{1}{c_{n}}\left(N_{p_{n}}-N_{p_{n}} * \mu_{p_{n}, C v}^{\prime}\right)$; alors on peut supposer que $\left(\eta_{n}\right)_{n=1}^{\infty}$ converge aussi vaguement vers une mesure de Radon positive $\eta$ lorsque $n \rightarrow \infty$. Remarquons que, pour $p>0$ et $q>0$ quelconques, $\operatorname{supp}\left(N_{p}\right)=\operatorname{supp}\left(N_{q}\right)$, car, pour $r>\max (p, q)$ quelconque, $N_{p}=\frac{1}{r-p} \sum_{n=1}^{\infty}\left((r-p) N_{r}\right)^{* n}$ et $N_{q}$ $=\frac{1}{r-q} \sum_{n=1}^{\infty}\left((r-q) N_{r}\right)^{* n}$. Donc, pour $p>0$ quelconque, $\operatorname{supp}(\eta)$ $\subset v \cap \operatorname{supp}\left(N_{p}\right)$ et $\int d \eta=1$. Le lemme de J. Deny (cité ci-dessus) donne que, pour $p>0$ quelconque,

$$
\lim _{n \rightarrow \infty} N_{p} * \mu_{p_{n}, C v}^{\prime}=N_{p} * \mu_{C v}^{\prime} \text { (vaguement), }
$$

et donc

$$
\begin{aligned}
\eta *\left(\varepsilon-p N_{p}\right) & =\lim _{n \rightarrow \infty} \frac{1}{c_{n}}\left(N_{p_{n}}-N_{p_{n}} * \mu_{p_{n}, C v}^{\prime}\right) *\left(\varepsilon-\left(p-p_{n}\right) N_{p}\right) \\
& =\lim _{n \rightarrow \infty} \frac{1}{c_{n}} N_{p} *\left(\varepsilon-\mu_{p_{n}, C v}^{\prime}\right)=0 \text { (vaguement) } .
\end{aligned}
$$

En faisant $p \rightarrow 0$, on arrive à $\eta=0$, d'où une contradiction. Donc $\left(N_{p}-N_{p} * \mu_{p, C v}^{\prime}\right)_{p>0}$ est vaguement bornée. Soient $0<p<q$ quelconques. Alors on a

$$
N_{q} * \mu_{p, C v}^{\prime}=N_{p} * \mu_{p, C v}^{\prime} *\left(\varepsilon-(q-p) N_{q}\right) \geqq N_{q}=N_{q} * \mu_{q, C v}^{\prime}
$$

dans $C v$. En rappelant la définition de $\mu_{p, C v}^{\prime}$, on a

$$
N_{q} * \mu_{p, C v}^{\prime} \geqq N_{q} * \mu_{q, C v}^{\prime} \text { dans } X
$$


Soient $\delta$ un nombre $>0$ et $f \in C_{K}^{+}$quelconques. Alors il existe un nombre $p_{0}>0$ tel que, pour $0<p<p_{0}$ et $0<q<p_{0}$ quelconques,

$$
\int f d\left(q N_{q}\right) *\left(N_{p}-N_{p} * \mu_{p, C v}^{\prime}\right)<\delta .
$$

Pour $p, q \in\left(0, p_{0}\right)$ vérifiant $p<q$ quelconques, on a

$$
\begin{gathered}
\int f d\left(N_{p}-N_{p} * \mu_{p, C v}^{\prime}\right)-\int f d\left(N_{q}-N_{q} * \mu_{q, C v}^{\prime}\right)-\delta \\
=(q-p) \int f d N_{q} *\left(N_{p}-N_{p} * \mu_{p, C v}^{\prime}\right)-\delta \\
-\int f d\left(N_{q} * \mu_{p, C v}^{\prime}-N_{q} * \mu_{q, C v}^{\prime}\right)<0 .
\end{gathered}
$$

Soient $\eta_{v, 1}$ et $\eta_{v, 2}$ deux points vaguement adhérents de $\left(N_{p}-N_{p} * \mu_{p, C v}^{\prime}\right)_{p>0}$ lorsque $p \rightarrow 0$ quelconques. Alors on a, pour tout $p \in\left(0, p_{0}\right)$,

$$
\int f d \eta_{v, j} \leqq \int f d\left(N_{p}-N_{p} * \mu_{p, C v}^{\prime}\right)+\delta \quad(j=1,2),
$$

et donc $\left|\int f d \eta_{v, 1}-\int f d \eta_{v, 2}\right| \leqq \delta$. En faisant $\delta \downarrow 0$, on arrive à l'egalite $\int f d \eta_{v, 1}=\int f d \eta_{v, 2}$, d'où $\eta_{v, 1}=\eta_{v, 2}$. Par conséquent, $\left(N_{p}-N_{p} * \mu_{p, C v}^{\prime}\right)_{p>0}$ converge vaguement lorsque $p \rightarrow 0$. L'égalité (2.22) résulte de l'équation résolvante et du lemme de J. Deny. La démonstration est ainsi complète.

On dit que tout le point vaguement adhérent de $\left(\mu_{p, C v}^{\prime}\right)_{p>0}$ lorsque $\mathrm{p} \rightarrow 0$ est une mesure balayée de $\varepsilon$ sur $C v$ relativement à $\left(N_{p}\right)_{p>0}$.

Proposition 16. Soit $N$ un noyau de convolution vérifiant $N(\{0\})>0$. Supposons qu'il existe un noyau de convolution $N^{\prime} \neq 0$ dans $S(N)$ tel que $N$ soit régulier par rapport à $N^{\prime}$. Soit $\mu^{\prime \prime}$ une mesure balayée transitivement de $\varepsilon$ sur $C\{0\}$ par rapport à $S(N)$ vérifiant $\mu^{\prime \prime}(\{0\})=0$. Alors

$$
N^{\prime} *\left(N-N * \mu^{\prime \prime}\right) \geqq 0 \text { dans } X \text {. }
$$

LEMme 17. Soient $N$ et $N^{\prime}$ deux noyaux de convolution et supposons que $N$ est régulier par rapport à $N^{\prime}$. Alors, pour une famille filtrante $\left(\mu_{\alpha}\right)_{\alpha \in A}$ des mesures de Radon positives convergeant vaguement vers une mesure de Radon positive $\mu, \lim _{\alpha} N * \mu_{\alpha}=N * \mu$ dès que $\left(N * \mu_{\alpha}\right)_{\alpha \in \Lambda}$ est vaguement bornée et que $N^{\prime} \geqq N^{\prime} * \mu_{\alpha}$ dans $X$. 
Démonstration du lemme 17. On peut supposer que $\left(N * \mu_{\alpha}\right)_{\alpha \in \Lambda}$ converge vaguement. Posons $\eta=\lim _{\alpha} N * \mu_{\alpha}$ (vaguement). Alors $\eta \geqq N * \mu$ dans $X$. Pour $\varphi \in C_{K}^{+}$vérifiant $0 \leqq \varphi \leqq 1$ quelconque, on a

$$
\eta-N * \mu=\lim _{\alpha} N *\left((1-\varphi)\left(\mu_{\alpha}-\mu\right)\right) \quad \text { (vaguement), }
$$

et donc, pour $v \in \mathscr{V}$ quelconque, il existe $\eta_{v} \in P\left(N, N^{\prime} ; v\right)$ tel que $\eta-N * \mu$ $\leqq \eta_{v}$ dans $X$. En faisant $v \uparrow X$, on arrive à $\eta=N * \mu$, d'où le lemme 17 .

Démonstration de la proposition 16. Comme $N^{\prime} \geqq N^{\prime} * \mu^{\prime \prime}$ dans $X$, pour $p>0$ quelconque, $N_{p}=\frac{1}{1+p}\left(\varepsilon+\sum_{n=1}^{\infty}\left(\frac{1}{1+p} \mu^{\prime \prime}\right)^{* n}\right)$ est défini et $\left(N_{p}\right)_{p>0}$ est une résolvante.

D'abord on suppose que $\lim _{\alpha} p N_{p}=0$. Comme $N^{\prime} \geqq p N^{\prime} * N_{p}$, le lemme 17 donne que $\lim _{p \rightarrow 0} N *\left(p N_{p}\right)^{\alpha}=0$ (vaguement). On a

$$
N=\lim _{p \rightarrow 0} N_{p} *\left(N-N * \mu^{\prime \prime}\right) \quad \text { (vaguement). }
$$

Donc $N \neq N * \mu^{\prime \prime}$. D'après (2.33), on peut écrire, pour une constante $c>0$ et pour une mesure de Radon positive $\sigma$, comme $N-N * \mu^{\prime \prime}$ $=c \varepsilon-\sigma$. Pour $v \in \mathscr{V}$ quelconque, posons $\sigma_{v}=\sigma$ sur $v$ et $\sigma_{v}=0$ dans $C v$. Soit $h \in C_{K}^{+}$vérifiant $N_{1} * h(x)>0$ sur $v+v$. Alors, pour $f \in C_{K}^{+}$portée par $v$ et un entier $n \geqq 1$ quelconque, il existe $0<p<1$ tel que

$$
N_{p} *\left(c \varepsilon-\sigma_{v}\right) * f+\frac{1}{n} N_{p} * h \geqq 0 \quad \operatorname{sur} \operatorname{supp}\left(\sigma_{v} * f\right) .
$$

Comme $N_{p} \sqsubset N^{\prime}$, on a

$$
N^{\prime} *\left(c \varepsilon-\sigma_{v}\right) * f+\frac{1}{n} N^{\prime} * h \geqq 0 \quad \text { sur } X .
$$

En faisant $n \rightarrow \infty, f \xi \rightarrow \varepsilon$ et ensuite $v \uparrow X$, on arrive à (2.31).

On suppose ensuite que $\lim _{p \rightarrow 0} p N_{p} \neq 0$. D'après la proposition 11, il existe un sous-groupe compact $\Gamma$ de $X$ tel que, pour $p>0$ quelconque, $\operatorname{supp}\left(N_{p}\right) \subset \Gamma$ et que $\lim _{p \rightarrow 0} p N_{p}=\xi_{\Gamma}$, où $\xi_{\Gamma}$ est la mesure de Haar normalisée de $\Gamma$. Alors, $\operatorname{supp}\left(\mu^{\prime \prime}\right) \subset \Gamma$ et

$$
\begin{aligned}
\left(N-N * \mu^{\prime \prime}\right) * \xi_{\Gamma} & =\lim _{p \rightarrow 0}\left(N-N * \mu^{\prime \prime}\right) *\left(p N_{p}\right) \\
& =\lim _{p \rightarrow 0} p N *\left(\varepsilon-p N_{p}\right)=0 \text { (vaguement). }
\end{aligned}
$$


Comme $N-N * \mu^{\prime \prime} \leqq 0$ dans $C\{0\}$, on a $\operatorname{supp}\left(N-N * \mu^{\prime \prime}\right) \subset \Gamma$. Donc on peut supposer que $\operatorname{supp}(N) \subset \Gamma$, et par suite on a (2.31). La démonstration est ainsi complète.

Par conséquent, on obtiendra la proposition suivante:

Proposition 18. Soit $N$ un noyau de convolution non-périodique vérifiant $N(\{0\})>0$. S'il existe un noyau de convolution $N^{\prime} \neq 0$ dans $S(N)$ tel que $N$ soit régulier par rapport à $N^{\prime}$, alors il existe deux semigroupes vaguement continus $\left(\alpha_{t}\right)_{t \geqq 0}$ et $\left(\beta_{t}\right)_{t \geqq 0}$ tels que, pour $t \in \boldsymbol{R}^{+}$quelconque, $N^{\prime} \geqq N^{\prime} * \alpha_{t}, N^{\prime} \geqq N^{\prime} * \beta_{t}, \frac{1}{t}\left(\varepsilon-\alpha_{t}\right), \frac{1}{t}\left(\varepsilon-\beta_{t}\right), \frac{1}{t}\left(N * \alpha_{t}-N\right)$ convergent vaguement lorsque $t \rightarrow 0, \lim _{p \rightarrow 0} p \int_{0}^{\infty} \exp (-p t) \alpha_{t} d t=0$ (vaguement) et que

$$
\lim _{t \rightarrow 0} \frac{1}{t} N *\left(\alpha_{t}-\varepsilon\right)=\lim _{t \rightarrow 0} \frac{1}{t}\left(\beta_{t}-\varepsilon\right) .
$$

Démonstration. Soit $\mu^{\prime \prime}$ une mesure balayée transitivement de $\varepsilon$ sur $C\{0\}$ par rapport à $S(N)$ vérifiant $\mu^{\prime \prime}(\{0\})=0$. Comme $N^{\prime} \geqq N^{\prime} * \mu^{\prime \prime}$, pour $t \in \boldsymbol{R}^{+}$quelconque,

$$
\alpha_{t}=\exp (-t)\left(\varepsilon+\sum_{n=1}^{\infty} \frac{t^{n}\left(\mu^{\prime \prime}\right)^{* n}}{n !}\right)
$$

a un sens et $\left(\alpha_{t}\right)_{t \geqq 0}$ est un semi-groupe vaguement continu. On a, pour $p>0$ quelconque, $N_{p}=\int_{0}^{\infty} \exp (-p t) \alpha_{t} d t$, où $\left(N_{p}\right)_{p>0}$ est la résolvante définie dans la démonstration de la proposition 16. Ecrivons, pour une constante $c \geqq 0$ et pour une mesure de Radon positive $\sigma$, comme $N-N * \mu^{\prime \prime}$ $=c \varepsilon-\sigma$. Montrons que $c>0$. Supposons contrairement que $c \leqq 0$. Alors (2.31) donne que $\sigma=0$, et donc $N-N * \mu^{\prime \prime}=0$. On a alors $\lim _{p \rightarrow 0} p N_{p} \neq 0$ (voir la démonstration de la proposition 16). Posons $\xi_{\Gamma}$ $\stackrel{p \rightarrow 0}{=} \lim _{p \rightarrow 0} p N_{p} ;$ alors $\operatorname{supp}\left(\mu^{\prime \prime}\right) \subset \operatorname{supp}\left(\xi_{\Gamma}\right)$ et $N * \xi_{\Gamma}=N * \xi_{\Gamma} * \mu^{\prime \prime}$, et donc $\int d \mu^{\prime \prime}=1$. L'égalité $N=N * \mu^{\prime \prime}$ et le théorème 1 dans [3] donnent que tout le point de $\operatorname{supp}\left(\mu^{\prime \prime}\right)$ est une période de $N^{(4)}$. Comme $\mu^{\prime \prime}(\{0\})=0$, ceci est en contradiction avec notre hypothèse. Par conséquent, $c>0$ et donc $N^{\prime} \geqq \frac{1}{c} N^{\prime} * \sigma$ (voir (2.31)). Pour $t \in \boldsymbol{R}^{+}$quelconque,

(4) On dit que $x \in X$ est une période de $N$ si $N=N * \varepsilon_{x}$. 


$$
\beta_{t}=\exp (-c t)\left(\varepsilon+\sum_{n=1}^{\infty} \frac{t^{n}(\sigma)^{* n}}{n !}\right)
$$

a un sens et $\left(\beta_{t}\right)_{t \geqq 0}$ est un semi-groupe vaguement continu. Comme $\lim _{t \rightarrow 0} \frac{1}{t}\left(\varepsilon-\alpha_{t}\right)=\varepsilon-\mu^{\prime \prime}$ et $\lim _{t \rightarrow 0} \frac{1}{t}\left(\varepsilon-\beta_{t}\right)=c \varepsilon-\sigma$, on voit (2.37). La démonstration est ainsi complète.

Soient $N$ un noyau de convolution et $\left(\alpha_{t}\right)_{t \geqq 0}$ un semi-groupe vaguement continu. Rappelons la définition de la sous-médianne conditionnelle de $N$ par rapport à $\left(\alpha_{t}\right)_{t \geqq 0}($ voir $\S 1)$. Lorsque $N_{0}=\int_{0}^{\infty} \alpha_{t} d t$ a un sens, cela est dit aussi conditionnellement sous-médian par rapport à $N_{0}$.

Proposition 19. Soient $N$ un noyau de convolution et $\left(N_{p}\right)_{p>0}$ une résolvante vérifiant $\lim _{p \rightarrow 0} p N_{p}=0$ (vaguement). Supposons que:

(a) Il existe un noyau de convolution $N^{\prime} \neq 0$ tel que $N=O\left(N^{\prime}\right)$, pour tout $p>0, N^{\prime} \geqq p N^{\prime} * N_{p}$ et que $N$ soit régulier par rapport à $N^{\prime}$.

(b) Il existe le semi-groupe vaguement continu $\left(\alpha_{t}\right)_{t \geqq 0}$ associé à $\left(N_{p}\right)_{p>0}$. Alors les trois énoncés suivants sont équivalents:

(1) $N$ est conditionnellement sous-médian par rapport à $\left(\alpha_{t}\right)_{t \geqq 0}$.

(2) Posons $\mathscr{D}^{+}\left(\lim _{p \rightarrow \infty} p N *\left(p N_{p}-\varepsilon\right)\right)$ l'ensemble des fonctions $f \in C_{K}^{+}$ vérifiant la condition que $p N *\left(p N_{p}-\varepsilon\right) * f$ converge uniformément sur tout compact lorsque $p \rightarrow \infty$. Alors $\mathscr{D}^{+}\left(\lim _{p \rightarrow \infty} p N *\left(p N_{p}-\varepsilon\right)\right)$ est dense dans $C_{K}^{+}$ et, pour $f \in \mathscr{D}^{+}\left(\lim _{p \rightarrow \infty} p N *\left(p N_{p}-\varepsilon\right)\right) \quad \grave{a}$ support $\subset C\{0\} \quad$ quelconque, $\lim _{p \rightarrow \infty} p N *\left(p N_{p}-\varepsilon\right) * f(0) \geqq 0$.

(3) Pour $v \in \mathscr{V}$ quelconque, $N * \mu_{C v}^{\prime} \geqq N$ dans $C v$, où $\mu_{C v}^{\prime}$ est la mesure balayée de $\varepsilon$ sur $C v$ relativement $\grave{a}\left(N_{p}\right)_{p>0}$.

Démonstration. $\quad(1) \Leftrightarrow(3)$ : Pour tout $p>0, N_{p} \prec N^{\prime}$, et donc $N^{\prime}$ $\geqq N^{\prime} * \mu_{C v}^{\prime}$. On voit alors que $N * \mu_{C v}^{\prime}$ a un sens, d'après $N=O\left(N^{\prime}\right)$. D'après (b), on voit facilement que, pour $t \in R^{+}$quelconque, $N^{\prime} \geqq N^{\prime} * \alpha_{t}$. Soit $\eta_{v}$ la même que dans la proposition 15. Alors on a $\eta_{v} \neq 0$, d'après la proposition 15. En utilisant le lemme de J. Deny, $\lim _{p \rightarrow 0} p N_{p} * \alpha_{t}=0$ (vaguement). On a donc, pour $t>0$ quelconque,

$$
\frac{1}{t} \eta_{v} *\left(\alpha_{t}-\varepsilon\right)=\lim _{p \rightarrow 0} \frac{1}{t}\left(\int_{0}^{\infty} \exp (-p t) \alpha_{s} d s\right) *\left(\varepsilon-\mu_{p, C v}^{\prime}\right) *\left(\alpha_{t}-\varepsilon\right)
$$




$$
\begin{aligned}
& =\lim _{p \rightarrow 0} \frac{1}{t}\left(\int_{0}^{t} \exp (-p t) \alpha_{s} d s-\frac{1-\exp (-p t)}{p}\left(p N_{p}\right) * \alpha_{t}\right) *\left(\mu_{p, C v}^{\prime}-\varepsilon\right) \\
& =\frac{1}{t}\left(\int_{0}^{t} \alpha_{s} d s\right) *\left(\mu_{C v}^{\prime}-\varepsilon\right) \quad \text { (vaguement) }
\end{aligned}
$$

où $\mu_{p, C v}^{\prime}$ est la mesure balayée de $\varepsilon$ sur $C v$ relativement à $N_{p}$. Ceci et le lemme 17 donnent que

$$
\lim _{t \rightarrow 0} \frac{1}{t} N * \eta_{v} *\left(\alpha_{t}-\varepsilon\right)=N *\left(\mu_{C v}^{\prime}-\varepsilon\right) \quad \text { (vaguement) . }
$$

Donc $\mathscr{D}^{+}\left(\lim _{t \rightarrow 0} \frac{1}{t}\left(N * \alpha_{t}-N\right)\right)$ contient le sous-cône convexe de $C_{K}^{+}$engendré par $\left\{f * \eta_{v} ; f \in C_{K}^{+}, v \in \mathscr{V}\right\}$, et par suite $\mathscr{D}^{+}\left(\lim _{t \rightarrow 0} \frac{1}{t}\left(N * \alpha_{t}-N\right)\right)$ est dense dans $\mathrm{C}_{K}^{+}$. Remarquons que, pour $f \in \mathscr{D}^{+}\left(\lim _{t \rightarrow 0} \frac{1}{t}\left(N * \alpha_{t}-N\right)\right)$ à support $\subset C\{0\}$ quelconque, $\left(\lim _{t \rightarrow 0} \frac{1}{t \int d \eta_{v}}\left(N * \alpha_{t}-N\right) * f * \eta_{v}(0)\right)_{v \in \mathcal{r}}$ converge vers $\lim _{t \rightarrow 0} \frac{1}{t}\left(N * \alpha_{t}-N\right) * f(0)$ lorsque $v \downarrow\{0\}$. Alors on voit facilement que (1) $\Leftrightarrow(3)$.

$(2) \Leftrightarrow(3)$ : De la même manière que ci-dessus, (2.22) donne que

$$
\lim _{p \rightarrow \infty} p N * \eta_{v} *\left(p N_{p}-\varepsilon\right)=N *\left(\mu_{C v}^{\prime}-\varepsilon\right) \quad \text { (vaguement) . }
$$

De la même manière, on voit que $(2) \Leftrightarrow(3)$. La démonstration est ainsi complète.

Dans ce cas, $\mathscr{D}^{+}\left(\lim _{t \rightarrow 0} \frac{1}{t}\left(N * \alpha_{t}-N\right)\right) \subset \mathscr{D}^{+}\left(\lim _{p \rightarrow \infty} p N *\left(p N_{p}-\varepsilon\right)\right)$ et il existe une mesure de Radon positive $\sigma$ en dehors de l'origine, et une seule telle que, pour $f \in \mathscr{D}^{+}\left(\lim _{t \rightarrow 0} \frac{1}{t}\left(N * \alpha_{t}-N\right)\right)$ à support $\subset C\{0\}$ quelconque,

$$
\int f d \sigma=\lim _{t \rightarrow 0} \frac{1}{t}\left(N * \alpha_{t}-N\right) * f(0)=\lim _{p \rightarrow \infty} p N *\left(p N_{p}-\varepsilon\right) * f(0)
$$

On a aussi que $\sigma=\lim _{v \downarrow\{0\}} \frac{1}{\int d \eta_{v}}\left(N * \mu_{C v}^{\prime}-\check{N}\right)$ (vaguement) en dehors de l'origine. 
En rappelant la présente démonstration, on voit la remarque suivante:

Remarque 20. Soient $N$ un noyau de convolution et $\left(\alpha_{t}\right)_{t \geqq 0}$ un semigroupe vaguement continu. Supposons qu'il existe un noyau de convolution $N^{\prime} \neq 0$ tel que, pour $t \in \boldsymbol{R}^{+}$quelconque, $N^{\prime} \geqq N^{\prime} * \alpha_{t}, N=O\left(N^{\prime}\right)$ et que $N$ soit régulier par rapport à $N^{\prime}$. Alors $\mathscr{D}^{+}\left(\lim _{t \rightarrow 0} \frac{1}{t}\left(N * \alpha_{t}-N\right)\right)$ est dense dans $C_{K}^{+}$.

En effet, de la même manière, (2.41) a lieu, et donc $\mathscr{D}^{+}\left(\lim _{t \rightarrow 0} \frac{1}{t}\left(N * \alpha_{t}-N\right)\right)$ est dense dans $\mathrm{C}_{K}^{+}$.

La remarque suivante résulte immédiatement de la définition de la sous-médianne conditionnelle.

Remarque 21. Soient $N$ un noyau de convolution et $\left(\alpha_{t}\right)_{t \geqq 0}$ un semigroupe vaguement continu. Alors pour que $N$ soit conditionnellement sous-médian par rapport à $\left(\alpha_{t}\right)_{t \geqq 0}$, il faut et il suffit que, pour $v \in \mathscr{V}$ quelconque, il existe une mesure de Radon positive $\eta_{v} \neq 0$ portée par $v$ telle que $\frac{1}{t}\left(N * \alpha_{t}-N\right) * \eta_{v}$ converge vaguement dans $X$ lorsque $t \rightarrow 0$ et que $\lim _{t \rightarrow 0} \frac{1}{t}\left(N * \alpha_{t}-N\right) * \eta_{v} \geqq 0$ en dehors de $v$.

La proposition suivante jouera un rôle dans la démonstration du théorème principal.

Proposition 22. Soient $N$ un noyau de convolution, $\left(\alpha_{t}\right)_{t \geqq 0}$ un semigroupe vaguement continu et $p$ une constante $>0$. Supposons qu'il existe un noyau de convolution $N^{\prime} \neq 0$ dans $S(N)$ tel que $N^{\prime} \geqq p \int_{0}^{\infty} \exp (-p t) N^{\prime} * \alpha_{t} d t$ et que $N$ soit régulier par rapport à $N^{\prime}$. Posons $N_{p}=\int_{0}^{\infty} \exp (-p t) \alpha_{t} d t$. Pour un ouvert $\omega$, on désigne par $\mu_{p, \omega}^{\prime}$ la mesure balayée de $\varepsilon$ sur $\omega$ relativement $\grave{a} N_{p}$. Si, pour un ouvert relativement compact $\omega$ quelconque, $N *\left(\varepsilon-p N_{p}\right) * \mu_{p, \omega}^{\prime} \geqq N *\left(\varepsilon-p N_{p}\right)$ dans $\omega$, alors $N$ est conditionnellement sous-médian par rapport à $\left(\alpha_{t}\right)_{t \geqq 0}$.

Démonstration. Soit $v \in \mathscr{V}$ quelconque. On choisit une famille filtrante à droite $\left(\omega_{\alpha}\right)_{\alpha \in A}$ des ouverts relativement compacts telle que $\bigcup_{\alpha \in A} \omega_{\alpha}=C v$. Alors $\mu_{p, C v}^{\prime}=\lim _{\alpha} \mu_{p, \omega_{\alpha}}^{\prime}$ et $N_{p} * \mu_{p, C v}^{\prime}=\lim _{\alpha} N_{p} * \mu_{p, \omega_{\alpha}}^{\prime}$ (vaguement). D'après l'inégalité $N^{\prime} \geqq p N^{\prime} * N_{p} * \mu_{p, \omega_{\alpha}}^{\prime}(\alpha \in \Lambda)$, le lemme 17 donne que 


$$
p N * N_{p} * \mu_{p, C v}^{\prime}=\lim _{\alpha} p N * N_{p} * \mu_{p, \omega_{\alpha}}^{\prime} \quad \text { (vaguement) . }
$$

Donc on a

$$
N *\left(\varepsilon-p N_{p}\right) * \mu_{p, C v}^{\prime} \geqq N *\left(\varepsilon-p N_{p}\right) \text { dans } C v .
$$

Posons $\eta_{p, v}=N_{p}-N_{p} * \mu_{p, C v}^{\prime}$; alors $\eta_{p, v} \neq 0$, d'après l'injectivité de $N_{p}$. D'après le lemme 17 , on a

$$
\begin{aligned}
N *(\varepsilon- & \left.p N_{p}\right) *\left(\mu_{p, C v}^{\prime}-\varepsilon\right) \\
= & \lim _{t \rightarrow 0} \frac{1}{t} N *\left(\varepsilon-p N_{p}\right) * \eta_{p, v} *\left(\exp (-p t) \alpha_{t}-\varepsilon\right) \\
= & \lim _{t \rightarrow 0}\left(\frac{1}{t} N * \eta_{p, v} *\left(\alpha_{t}-\varepsilon\right)-\frac{1}{t}(1-\exp (-p t)) N * \eta_{p, v} * \alpha_{t}\right. \\
& \left.\quad+\frac{1}{t} p N * N_{p} * \eta_{p, v} *\left(\varepsilon-\exp (-p t) \alpha_{t}\right)\right) \\
= & \lim _{t \rightarrow 0} \frac{1}{t} N * \eta_{p, v} *\left(\alpha_{t}-\varepsilon\right) .
\end{aligned}
$$

D'après la remarque $21, N$ est conditionnellement sous-médian par rapport à $\left(\alpha_{t}\right)_{t \geqq 0}$. La démonstration est ainsi complète.

Considérons la convergence d'une famille filtrante des résolvante.

Proposition 23. Soient $\left(\left(N_{p, \alpha}\right)_{p>0}\right)_{\alpha \in \Lambda}$ une famille filtrante des résolvantes des noyaux de convolution et $N^{\prime}$ un noyau de convolution non-zéro. Supposons que, pour $p>0$ et $\alpha \in \Lambda$ quelconques, $N^{\prime} \geqq p N^{\prime} * N_{p, \alpha}$. Si, pour $p_{0}>0,\left(N_{p_{0}, \alpha}\right)_{\alpha \in \Lambda}$ et $\left(N^{\prime} * N_{p_{0}, \alpha}\right)_{\alpha \in \Lambda}$ convergent vaguement et si $\lim _{\alpha} N^{\prime} * N_{p_{0}, \alpha}$ $=N^{\prime} *\left(\lim _{\alpha} N_{p_{0}, \alpha}\right)$, alors, pour $p>0$ quelconque, $\left(N_{p, \alpha}\right)_{\alpha \in A}$ converge vaguement aussi. Posons $N_{p}=\lim _{\alpha} N_{p, \alpha}(p>0)$. Alors $\left(N_{p}\right)_{p>0}$ est une résolvante et, pour $p>0$ quelconque, $\left(N^{\prime} * N_{p, \alpha}\right)_{\alpha \in A}$ converge vaguement vers $N^{\prime} * N_{p}$.

Démonstration. Posons $N_{p_{0}}=\lim _{\alpha} N_{p_{0}, \alpha}$. Soit $\quad p>0$ quelconque. Comme, pour tout $\alpha \in \Lambda, N^{\prime} \geqq p N^{\prime} * N_{p, \alpha},\left(N_{p, \alpha}\right)_{\alpha \in A}$ est vaguement bornée. Soient $N_{p}$ et $N_{p}^{\prime}$ deux points vaguement adhérents de $\left(N_{p, \alpha}\right)_{\alpha \in A}$ quelconques. On choisit deux sous-familles filtrantes $\left(N_{p, \alpha^{\prime}}\right)_{\alpha^{\prime} \in A^{\prime}}$ et $\left(N_{p, \alpha^{\prime \prime}}\right)_{\alpha^{\prime \prime} \in A^{\prime \prime}}$ de $\left(N_{p, \alpha}\right)_{\alpha \in \Lambda}$ qui convergent vaguement vers $N_{p}$ et $N_{p}^{\prime}$, respectivement. Soit $f \in C_{K}^{+}$quelconque. Comme $N_{p, \alpha} \prec N^{\prime}$ (voir la proposition 10), on peut supposer qu'il existe $g \in C_{K}^{+}$telle que, pour $\alpha \in \Lambda$ quelconque, $N_{p, \alpha} * f$ $\leqq N^{\prime} * g$ sur $X$. Alors on a 


$$
\begin{aligned}
N_{p} * N_{p_{0}} * f(0) & \leqq \varlimsup_{\lim _{\alpha^{\prime}}} N_{p, \alpha^{\prime}} * N_{p_{0}, \alpha^{\prime}} * f(0) \leqq \varlimsup_{\alpha^{\prime}} N_{p, \alpha^{\prime}} * N_{p_{0}, \alpha^{\prime}} * f(0) \\
& =\varlimsup_{\alpha^{\prime}}\left(N^{\prime} * N_{p_{0}, \alpha^{\prime}} * g(0)-N_{p_{0}, \alpha^{\prime}} *\left(N^{\prime} * g-N_{p, \alpha^{\prime}} * f\right)(0)\right) \\
& \leqq N^{\prime} * N_{p_{0}} * g(0)-N_{p_{0}} *\left(N^{\prime} * g-N_{p} * f\right)(0) \\
& =N_{p} * N_{p_{0}} * f(0) .
\end{aligned}
$$

Comme $f$ est quelconque, $N_{p} * N_{p_{0}}=\lim _{\alpha^{\prime}} N_{p, \alpha^{\prime}} * N_{p_{0}, \alpha^{\prime}}$ (vaguement). De la même manière, on a $N_{p}^{\prime} * N_{p_{0}}=\lim _{\alpha^{\prime \prime}} N_{p, \alpha^{\prime \prime}} * N_{p_{0}, \alpha^{\prime \prime}}$ (vaguement). Donc

$$
N_{p_{0}}=N_{p} *\left(\varepsilon-\left(p_{0}-p\right) N_{p_{0}}\right)=N_{p}^{\prime} *\left(\varepsilon-\left(p_{0}-p\right) N_{p_{0}}\right) .
$$

Si $\left|p_{0}-p\right|<p_{0}$, alors $\sum_{n=1}^{\infty}\left(\left(p_{0}-p\right) N_{p_{0}}\right)^{* n}$ converge vaguement et

$$
N_{p}=N_{p}^{\prime}=N_{p_{0}} *\left(\varepsilon+\sum_{n=1}^{\infty}\left(\left(p_{0}-p\right) N_{p_{0}}\right)^{* n}\right),
$$

car $N^{\prime} \geqq p_{0} N^{\prime} * N_{p_{0}}$. On voit ainsi que, pour $p \in\left(0,2 p_{0}\right)$ quelconque, $\left(N_{p, \alpha}\right)_{\alpha \in \Lambda}$ converge vaguement. On voit encore, par récurrence, que, pour $p>0$ quelconque, $\left(N_{p, \alpha}\right)_{\alpha \in \Lambda}$ converge vaguement. En remarquant (2.47), on voit que $\left(N_{p}\right)_{p>0}$ est une résolvante. De la même manière, on voit que, pour $p \geqq p_{0}$ quelconque, $\left(N^{\prime} * N_{p, \alpha}\right)_{\alpha \in \Lambda}$ converge vaguement vers $N^{\prime} * N_{p}$. En remarquant que, pour $\alpha \in \Lambda$ et un entier $n \geqq 1$ quelconques, $N^{\prime} \geqq N^{\prime} *\left(p_{0} N_{p_{0}}\right)^{* n}$, on voit, par récurrence, que, pour un entier $n \geqq 1$ quelconque,

$$
\lim _{\alpha} N^{\prime} *\left(p_{0} N_{p_{0}, \alpha}\right)^{* n}=N^{\prime} *\left(p_{0} N_{p_{0}}\right)^{* n} \quad \text { (vaguement) . }
$$

Soient $0<p<p_{0}$ et $f \in C_{K}^{+}$quelconques. Alors, pour un entier $k \geqq 1$ quelconque,

$$
\begin{gathered}
\int f d N^{\prime} * N_{p} \leqq \underline{\lim } \int f d N^{\prime} * N_{p, \alpha} \leqq \varlimsup_{\alpha} \int f d N^{\prime} * N_{p, \alpha} \\
=\varlimsup_{\alpha}\left(\frac{1}{p_{0}-p} \int f d N^{\prime} * \sum_{n=1}^{\infty}\left(\left(p_{0}-p\right) N_{p_{0}, \alpha}\right)^{* n}\right) \\
\leqq \int f d N^{\prime} * N_{p}+\sum_{n=k}^{\infty}\left(\frac{p_{0}-p}{p_{0}}\right)^{n} \int f d N^{\prime} .
\end{gathered}
$$

On voit ainsi que $\left(N^{\prime} * N_{p, \alpha}\right)_{\alpha \in \Lambda}$ converge vaguement vers $N^{\prime} * N_{p}$. La démonstration est ainsi complète.

Proposition 24. Soient $N$ un noyau de convolution vérifiant $N \prec N$, 
$N^{\prime} \neq 0$ un noyau de convolution et $p>0$ une constante. Si $N^{\prime} \geqq p N^{\prime} * N$, $N^{\prime} \neq p N^{\prime} * N$ et $N \prec N^{\prime} *(\varepsilon-p N)$, alors il existe un noyau de convolution $N_{0}$ et la résolvante $\left(N_{q}\right)_{q \geqq 0}$ associée d̀ $N_{0}$ telle que $N_{p}=N$. Dans ce cas, $N_{0}$ est déterminé d'une manière unique et $N_{0} \prec N^{\prime}$.

Démonstration. Comme $N^{\prime} \geqq p N^{\prime} * N$ et $N^{\prime} \neq p N^{\prime} * N, \sum_{n=1}^{\infty}(p N)^{* n}$ converge vaguement, et donc, en utilisant le lemme de J. Deny, $\bigcap_{v \in \mathscr{r}} P(N ; v)$ $=\{0\}$; c'est-à-dire, $N$ est régulier. D'après la remarque 9 , il existe la résolvante $\left(M_{q}\right)_{q \geqq 0}$ associée à $N$. D'après $N \prec N^{\prime} *(\varepsilon-p N)$, on a, pour tout $q>0$,

$$
q N^{\prime} *(\varepsilon-p N) * M_{q} \leqq N^{\prime} *(\varepsilon-p N),
$$

et donc $(p+q) N^{\prime} * M_{q} \leqq N^{\prime}$. Posons, pour $q \in[0, p)$ quelconque, $N_{q}$ $=\frac{1}{p-q} \sum_{n=1}^{\infty}\left((p-q) M_{0}\right)^{* n}$ et, pour $q \in[p, \infty)$ quelconque, $N_{q}=M_{q-p}$. Alors, pour $q \geqq 0$ quelconque,

$$
N_{q}-N_{p}=(p-q) N_{p} * N_{q} \text { et } \lim _{q \rightarrow 0} N_{q}=N_{0} .
$$

Donc, pour $q \geqq 0$ et $q^{\prime}>0$ quelconques,

$$
\begin{aligned}
N_{q}-N_{q^{\prime}}= & (p-q)\left(N_{q^{\prime}}+\left(q^{\prime}-p\right) N_{p} * N_{q^{\prime}}\right) * N_{q} \\
& -\left(p-q^{\prime}\right)\left(N_{q}+(q-p) N_{p} * N_{q}\right) * N_{q^{\prime}} \\
= & \left(q^{\prime}-q\right) N_{q} * N_{q^{\prime}} .
\end{aligned}
$$

D'après (2.53) et (2.54), $\left(N_{q}\right)_{q \geqq 0}$ est la résolvante associée à $N_{0}$. L'unicité de $N_{0}$ est bien connue. On a, pour $q>0, N^{\prime} \geqq q N^{\prime} * N_{q}$, et donc la proposition 10 donne que, pour $q>0$ quelconque, $N_{q} \prec N^{\prime}$. En faisant $q \downarrow 0$, on arrive à $N_{0} \prec N^{\prime}$. La démonstration est ainsi complète.

\section{§3. Le principe de domination relatif et la sous-médianne con- ditionnelle}

Dans ce paragraphe, on supposera toujours qu'il n'existe aucun sousgroupe compact de $X$ excepté $\{0\}$.

LEMME 25. Soit $\left(N_{p}\right)_{p>0}$ une résolvante des noyaux de convolution $\neq 0$. Alors il existe le semi-groupe vaguement continu associé $\grave{a}\left(N_{p}\right)_{p>0}$.

Démonstration. Pour $p>0$ et $v \in \mathscr{V}$, on désigne par $\mu_{p, C v}^{\prime}$ une mesure balayée de $\varepsilon$ sur $C v$ relativement à $N_{p}$. Comme, pour $0<q<p$, on a 
$N_{q} \geqq N_{q} * \mu_{p, C v}^{\prime}$, le lemme de J. Deny donne que $\lim _{v \uparrow X} N_{p} * \mu_{p, C v}^{\prime}=0$ (vaguement). Donc il existe $v \in \mathscr{V}$ tel que $N_{p} \neq N_{p} * \mu_{p, c v}^{\prime}$. D'après $N_{p} \prec N_{p}$, $\operatorname{supp}\left(N_{p}-N_{p} * \mu_{p, C v}^{\prime}\right) \ni 0$. Soit $\Gamma$ l'ensemble des périodes de $N_{p}$; alors $\Gamma$ est un sous-groupe fermé de X. Comme tout le point de $\Gamma$ est aussi une période de $N_{p}-N_{p} * \mu_{p, C v}^{\prime}$, on a $\Gamma \subset v$. En vertu de notre hypothèse pour $X$, on a $\Gamma=\{0\}$. Donc la proposition 13 donne le présent lemme.

Montrons le premier théorème principal.

ThÉoRÈme 26. Soient $N$ un noyau de convolution et $N^{\prime}$ un noyau de convolution $\neq 0$. Supposons que $N$ est régulier par rapport à $N^{\prime}$.

Alors les deux énoncés suivants sont équivalents:

(a) $N$ vérifie le principe de domination relatif à $N^{\prime}$.

(b) $N=O\left(N^{\prime}\right)$ et il existe un semi-groupe vaguement continu $\left(\alpha_{t}\right)_{t \geqq 0}$ vérifiant $\alpha_{t} \neq \varepsilon(t>0)$ tel que $N$ soit conditionnellement sous-médian par rapport à $\left(\alpha_{t}\right)_{t \geqq 0}$ et que, pour $t \geqq 0$ quelconque, $N^{\prime} \geqq N^{\prime} * \alpha_{t}$.

Démonstration. (b) $\Rightarrow$ (a): On peut supposer que $N \neq 0$. Comme $N$ est régulier par rapport à $N^{\prime}, N^{\prime} \neq 0$. Posons, pour $p>0$ quelconque, $N_{p}=\int_{0}^{\infty} \exp (-p t) \alpha_{t} d t$; alors, $N^{\prime} \geqq p N^{\prime} * N_{p}$. Montrons que $\lim _{p \rightarrow 0} p N_{p}=0$ (vaguement). Supposons contrairement que cette égalité n'a pas lieu. Alors, d'après la proposition 11 et notre hypothèse pour $X, \lim _{p \rightarrow 0} p N_{p}=\varepsilon$ (vaguement) et, pour tout $p>0, \operatorname{supp}\left(N_{p}\right) \subset\{0\}$, et $\operatorname{donc} \operatorname{supp}\left(\alpha_{t}\right) \subset\{0\}$. Comme $N^{\prime} \geqq N^{\prime} * \alpha_{t}$ et $\alpha_{t} \neq \varepsilon(t>0)$, il existe une constante $a>0$ telle que $\alpha_{t}=\exp (-a t) \varepsilon$. Alors $\lim _{p \rightarrow 0} p N_{p}=\lim _{p \rightarrow 0} \frac{p}{p+a}=0$, d'où une contradiction. On obtient ainsi que $\lim _{p \rightarrow 0} p N_{p}=0$ (vaguement). D'après le lemme 17, on a aussi $\lim _{p \rightarrow 0} N *\left(p N_{p}\right)=0$ (vaguement). Pour montrer que $N \prec N^{\prime}$, il suffit de montrer que, pour $f, g \in C_{K}^{+}$quelconques,

$$
N * f<N^{\prime} * g \operatorname{sur} \operatorname{supp}(f) \Rightarrow N * f \leqq N^{\prime} * g \operatorname{sur} X .
$$

On choisit $v_{0} \in \mathscr{V}$ tel que, pour une mesure de Radon positive $\nu$ portée par $v_{0}$ de masse totale d'unité quelconque,

$$
N * \nu * f(x)<N^{\prime} * g(x) \text { sur } \operatorname{supp}(f)+v_{0},
$$

où $\operatorname{supp}(f)+v_{0}=\left\{x+y ; x \in \operatorname{supp}(f), y \in v_{0}\right\}$. Pour $v \in \mathscr{V}$, on désigne par $\mu_{p, C v}^{\prime}$ la mesure balayée de $\varepsilon$ sur $C v$ relativement à $N_{p}$. Posons 
$\mu_{C v}^{\prime}=\lim _{p \rightarrow 0} \mu_{p, C v}^{\prime}$ et $\eta_{v}=\lim _{p \rightarrow 0}\left(N_{p}-N_{p} * \mu_{p, C v}^{\prime}\right)$ (voir la proposition 15). D'après la proposition 15 , on a $\eta_{v} \neq 0$. D'après la proposition 10 , on a $N_{p} \prec N^{\prime}$. En utilisant la proposition 2, on voit qu'il existe $h_{p} \in C_{K}^{+}$vérifiant $N_{p} * h_{p}$ $=N^{\prime} * g \operatorname{sur} \operatorname{supp}(f)+v_{0}$ et $N_{p} * h_{p} \leqq N^{\prime} * g \operatorname{sur} X$. Pour $p>0$ et $v \in \mathscr{V}$ vérifiant $v \subset v_{0}$ quelconques, on pose

$$
\lambda_{v, 1}=\frac{1}{\int d \eta_{v}}\left(N-N * \mu_{C v}^{\prime}\right)^{+} \quad \text { et } \quad \lambda_{v, 2}=\frac{1}{\int d \eta_{v}}\left(N-N * \mu_{C v}^{\prime}\right)^{-} .
$$

Alors, d'après la proposition 19 , on a $\operatorname{supp}\left(\lambda_{v, 1}\right) \subset v$. D'après la proposition 15 , on a

$$
\begin{aligned}
& N_{p} * \lambda_{v, 1} * f-N_{p} * \lambda_{v, 2} * f \\
& \quad=\frac{1}{\int d \eta_{v}} N * \eta_{v} *\left(\varepsilon-p N_{p}\right) * f<N_{p} * h_{p} \\
& \quad \operatorname{sur} \operatorname{supp}(f)+v_{0} \supset \operatorname{supp}\left(f * \lambda_{p, v, 1}\right) .
\end{aligned}
$$

En utilisant $N_{p} \prec N_{p}$, on a

$$
\frac{1}{\int d \eta_{v}} N * \eta_{v} *\left(\varepsilon-p N_{p}\right) * f \leqq N_{p} * h_{p} \leqq N^{\prime} * g \text { sur } X
$$

En faisant $v \downarrow\{0\}$ et ensuite $p \rightarrow 0$, on arrive à $N * f \leqq N^{\prime} * g$ sur $X$, d'où (3.1). On voit ainsi que (b) $\Rightarrow$ (a).

(a) $\Rightarrow$ (b): D'après la proposition 18, il suffit de montrer (a) $\Rightarrow$ (b) dans le cas où $N(\{0\})=0$. Soit a une constante $>0$ quelconque. On remarque que $S(N+a \varepsilon) \subset S(N)$ et que $N+a \varepsilon$ est régulier par rapport à $N^{\prime}$. Soit $\mu_{a}^{\prime \prime}$ une mesure balayée transitivement de $\varepsilon$ sur $C\{0\}$ relativement à $\left(N+a \varepsilon, N^{\prime}\right)$ vérifiant $\mu_{a}^{\prime \prime}(\{0\})=0$. D'après le corollaire 4 et la proposition 18, il existe deux semi-groupes vaguement continus $\left(\alpha_{a, t}\right)_{t \geq 0}$ et $\left(\beta_{a, t}\right)_{t \geqq 0}$ tels que l'on ait:

$$
\begin{gathered}
\alpha_{a, t}=\exp (-t)\left(\varepsilon+\sum_{n=1}^{\infty} \frac{t^{n}\left(\mu_{a}^{\prime \prime}\right)^{n}}{n !}\right) . \\
\lim _{t \rightarrow 0} \frac{1}{t}\left(\beta_{a, t}-\varepsilon\right)=(N+a \varepsilon) *\left(\mu_{a}^{\prime \prime}-\varepsilon\right) \text { (vaguement). }
\end{gathered}
$$

On a, pour $t \in R^{+}$quelconque, $N^{\prime} \geqq N^{\prime} * \alpha_{a, t}$. Soient $\varphi \neq 0 \in C_{K}^{+}$quelconque fixée et $x_{0} \in X$ vérifiant $\varphi\left(x_{0}\right)>0$ fixé. Posons, pour toute $c>0$, 
$g_{c}=\left(\int_{0}^{\infty} \exp (-t) \alpha_{a, c t} d t\right) * \varphi$. Alors $\left(g_{c}\right)_{c>0}$ converge uniformément vers $\varphi$ sur tout compact lorsque $c \rightarrow 0$ et vers 0 sur tout compact lorsque $c \rightarrow \infty$, car

$$
g_{c}=\left(\frac{1}{c} \int_{0}^{\infty} \exp \left(-\frac{1}{c} t\right) \alpha_{a, t} d t\right) * \varphi
$$

$\left(\int_{0}^{\infty} \exp (-p t) \alpha_{a, t} d t\right)_{p>0}$ est une résolvante et $\operatorname{supp}\left(\alpha_{t}\right)$ n'est pas compact. dès que $\mu_{a}^{\prime \prime} \neq 0(t>0)$. Il existe donc une constante $c_{a}>0$ telle que $g_{c_{a}}\left(x_{0}\right)=\frac{1}{2} \varphi\left(x_{0}\right)$. Pour tout $p>0$, on pose $N_{p, a}=\int_{0}^{\infty} \exp (-p t) \alpha_{a, c_{a} t} d t$. Alors $\left(N_{p, a}\right)_{p>0}$ est une résolvante et, pour $p>0$ quelconque, $N^{\prime}$ $\geqq p N^{\prime} * N_{p, a}$. Donc, pour $p>0$ quelconque, $\left(N_{p, a}\right)_{a>0}$ est vaguement bornée. On peut supposer que $\left(N_{1, a}\right)_{a>0}$ converge vaguement lorsque $a \rightarrow 0$. Posons $N_{1}=\lim _{a \rightarrow 0} N_{1, a}$. Alors $N_{1} * \varphi\left(x_{0}\right)=\frac{1}{2} \varphi\left(x_{0}\right)$, et donc $N_{1} \neq 0$. On a aussi $N_{1} \prec N_{1}$ et $N^{\prime} \geqq N^{\prime} * N_{1}$. Comme $N_{1, a} \prec N^{\prime}$ et $N_{1, a} \prec N^{\prime} *\left(\varepsilon-N_{1, a}\right)$, on a $N_{1} \prec N^{\prime}$ et, pour $f \in C_{K}^{+}$vérifiant $f \leqq 1$ quelconque, $N_{1} \prec N^{\prime} *\left(\varepsilon-f N_{1}\right)$. En faisant $f \uparrow 1$, on a $N_{1} \prec N^{\prime} *\left(\varepsilon-N_{1}\right)$. Montrons qu'il existe une résolvante $\left(M_{p}\right)_{p>0}$ des noyaux de convolution $\neq 0$ vérifiant $M_{1}=N_{1}$. Supposons d'abord que $N^{\prime}=N^{\prime} * N_{1}$. Alors $N^{\prime} * N_{1}=\lim _{a \rightarrow 0} N^{\prime} * N_{1, a}$ (vaguement). D'après la proposition 23 , on voit que, pour $p>0$ quelconque, $\left(N_{p, a}\right)_{a>0}$ converge vaguement lorsque $a \rightarrow 0$ et que, en posant $N_{p}$ $=\lim _{a \rightarrow 0} N_{p, a},\left(N_{p}\right)_{p>0}$ est une résolvante. On a aussi $N^{\prime}=p N^{\prime} * N_{p}$, et donc $N_{p} \neq 0$. Par conséquent $\left(N_{p}\right)_{p>0}$ est une résolvante demandée. Supposons ensuite que $N^{\prime} \neq N^{\prime} * N_{1}$. Alors, d'après la proposition 24, il existe une résolvante $\left(M_{p}\right)_{p>0}$ des noyaux de convolution $\neq 0$, et une seule telle que $M_{1}=N_{1}$. Soit $\left(\alpha_{t}\right)_{t \geqq 0}$ le semi-groupe vaguement continu associé à $\left(M_{p}\right)_{p>0}$. Comme $N_{1} * \varphi\left(x_{0}\right)=\frac{1}{2} \varphi\left(x_{0}\right), N_{1} \neq \varepsilon$, et donc, pour tout $t>0, \alpha_{t} \neq \varepsilon$. Montrons finalement que $N$ est conditionnellement sous-médian par rapport à $\left(\alpha_{t}\right)_{t \geqq 0}$. Soit $\omega$ un ouvert relativement compact dans $X$ quelconque. On choisit une famille filtrante à droite $\left(\omega_{\alpha}\right)_{\alpha \in A}$ des ouverts telle que $\bar{\omega}_{\alpha} \subset \omega$ et $\bigcup_{\alpha \in A} \omega_{\alpha}=\omega$. Pour $a>0$, on désigne par $\mu_{\omega_{\alpha}, a}^{\prime}$ la mesure balayée de $\varepsilon$ sur $\omega_{\alpha}$ relativement à $N_{1, a}$. Comme $N_{1, a} \prec N^{\prime}, N^{\prime} \geqq N^{\prime} * \mu_{\omega_{\alpha}, a}^{\prime}$, et donc $\left(\mu_{\omega_{\alpha}, a}^{\prime}\right)_{a>0}$ est vaguement bornée. On a

$$
\begin{aligned}
& (N+a \varepsilon) *\left(\varepsilon-N_{1, a}\right) *\left(\mu_{\omega_{\alpha}, a}^{\prime}-\varepsilon\right) \\
& \quad=c_{a}(N+a \varepsilon) *\left(\varepsilon-\mu_{a}^{\prime \prime}\right) * N_{1, a} *\left(\mu_{\omega_{\alpha}, a}^{\prime}-\varepsilon\right) \geqq 0 \text { dans } \omega_{\alpha} .
\end{aligned}
$$


Soit $\mu_{\omega_{\alpha}}^{\prime}$ un point vaguement adhérent de $\left(\mu_{\omega_{\alpha}, a}^{\prime}\right)_{a>0}$ lorsque $a \downarrow 0$. D'après le lemme 17 et (3.8), on a

$$
N *\left(\varepsilon-N_{1}\right) *\left(\mu_{\omega_{\alpha}}^{\prime}-\varepsilon\right) \geqq 0 \text { dans } \omega_{\alpha} .
$$

On a aussi $\operatorname{supp}\left(\mu_{\omega_{\alpha}}^{\prime}\right) \subset \bar{\omega}_{\alpha}, N_{1} * \mu_{\omega_{\alpha}}^{\prime} \leqq N_{1}$ dans $X$ et $N_{1} * \mu_{\omega_{\alpha}}^{\prime}=N_{1}$ dans $\omega_{\alpha}$. Soit $\mu_{\omega}^{\prime}$ la mesure balayée de $\varepsilon$ sur $\omega$ relativement à $N_{1}$. Alors $\mu_{\omega}^{\prime}$ est un point vaguement adhérent de $\left(\mu_{\omega_{\alpha}}^{\prime}\right)_{\alpha \in A}$ lorsque $\omega_{\alpha} \uparrow \omega$, et donc

$$
N *\left(\varepsilon-N_{1}\right) *\left(\mu_{\omega}^{\prime}-\varepsilon\right) \geqq 0 \text { dans } \omega .
$$

D'après la proposition 22, $N$ est conditionnellement sous-médian par rapport à $\left(\alpha_{t}\right)_{t \geqq 0}$. Comme, pour tout $p>0, N^{\prime} \geqq p N^{\prime} * M_{p}$, on a, pour $t \in \boldsymbol{R}^{+}$quelconque, $N^{\prime} \geqq N^{\prime} * \alpha_{t}$. On voit ainsi (a) $\Rightarrow(\mathrm{b})$. La démonstration est complète.

En particulier, on aura corollaire suivant:

CoRollaire 27. Soit $N$ un noyau de convolution régulier par rapport $\grave{a} \xi$. Alors les deux énoncés suivants sont équivalents:

(a) $N$ vérifie le principe classique du maximum; c'est-à-dire, $N \prec \xi$.

(b) $N$ est borné( $e^{(5)}$ et il existe un semi-groupe vaguement continu sousmarkovien $\left(\alpha_{t}\right)_{t \geqq 0}$ vérifiant $\alpha_{t} \neq \varepsilon(t>0)$ tel que $N$ soit conditionnellement sous-médian par rapport à $\left(\alpha_{t}\right)_{t \geqq 0}$.

On dit que $\left(\alpha_{t}\right)_{t \geqq 0}$ est sous-markovien si, pour tout $t \geqq 0, \int d \alpha_{t} \leqq 1$.

En regardant la démonstration du théorème 26 , on verra la remarque suivante:

Remarque 28. Soit $N$ un noyau de convolution. Supposons qu'il existe $N^{\prime} \neq 0 \in S(N)$ tel que $N$ soit régulier par rapport à $N^{\prime}$. Alors il existe un semi-groupe vaguement continu $\left(\alpha_{t}\right)_{t \geqq 0}$ vérifiant $\alpha_{t} \neq \varepsilon(t>0)$ tel que $N$ soit conditionnellement sous-médian par rapport à $\left(\alpha_{t}\right)_{t \geqq 0}$ et que, pour $N^{\prime \prime} \in S(N)$ et $t \in R^{+}$quelconques, $N^{\prime \prime} \geqq N^{\prime \prime} * \alpha_{t}$.

Rappelons la démonstration du théorème 26. Soit a une constante $>0$ quelconque. Soient $\mu_{a}^{\prime \prime}$ une mesure balayée transitivement de $\varepsilon$ sur $C\{0\}$ par rapport à $S(N+a \varepsilon)$ vérifiant $\mu_{a}^{\prime \prime}(\{0\})=0$ et $\left(\alpha_{a, t}\right)_{t \geqq 0},\left(\beta_{a, t}\right)_{t \geqq 0}$ les deux semi-groupes vaguement continus définis par $\mu_{a}^{\prime \prime}$ de la même manière que dans le théorème 26. En faisant la même discussion comme dans

(5) Cela signifie que, pour $f \in C_{K}$ quelconque, $N * f$ est bornée sur $X$. 
le théorème 26 concernant la famille $\left(\left(\alpha_{a, t}\right)_{t \geqq 0}\right)_{a>0}$ des semi-groupes vaguement continus, on voit facilement qu'il existe un semi-groupe vaguement continu $\left(\alpha_{t}\right)_{t \geqq 0}$ demandé.

Question 29. Est-ce que $\left(\alpha_{t}\right)_{t \geqq 0}$ dans la remarque 28 est toujours unique excepté la multiplication constante?

Soient $\left(\alpha_{t}\right)_{t \geqq 0}$ et $\left(\beta_{t}\right)_{t \geqq 0}$ deux semi-groupes vaguement continus. On dit que $\left(\beta_{t}\right)_{t \geqq 0}$ est un multiple constant de $\left(\alpha_{t}\right)_{t \geqq 0}$ si, pour $t \in \boldsymbol{R}^{+}$quelconque, $\beta_{t}=\alpha_{c t}$, où $c$ est une constante $>0$ indépendante de $t$.

Dans le thérème 26, $\left(\alpha_{t}\right)_{t \geqq 0}$ n'est pas toujours unique excepté la multiplication constante. Par exemple, considérer les noyaux de RieszFrostman dans $\boldsymbol{R}^{n}$.

Le théorème suivant indique que le principe classique du maximum et le principe de domination relatif au noyau de convolution de Hunt sont. essentiels.

ThÉoRème 30. Soit $N$ un noyau de convolution vérifiant $S(N) \neq \emptyset$ et $S(N) \neq\{0\}$. Supposons que le sous-groupe fermé engendré par supp $(N)$ est égal à $X$ et qu'il existe $N^{\prime} \neq 0 \in S(N)$ tel que $N$ soit régulier par rapport à $N$. Alors on a (a) ou bien (b).

(a) Il existe une fonction exponentielle $\varphi(x)>0$ sur $X$ telle que $S(N)=\left\{a \varphi \xi ; a \in \boldsymbol{R}^{+}\right\}$.

(b) Il existe un noyau de convolution de Hunt $N_{0}$ tel que $N$ soit conditionnellement sous-médian par rapport à $N_{0}$ et que

$$
S(N)=\left\{N_{0} * \lambda+\eta ; \lambda \in D^{+}\left(N_{0}\right), \eta \text { est } \geqq 0 \text { et } N_{0} \text {-harmonique }\right\} \text {. }
$$

Dans (b), $N_{0}$ est uniquement déterminé excepté la multiplication constante.

On dit qu'une fonction finie et continue $\varphi$ est exponentielle si, pour $x, y \in X$ quelconques, $\varphi(x+y)=\varphi(x) \varphi(y)$. On voit évidemment que $S(N)$ $=\left\{a \varphi \xi ; a \in \boldsymbol{R}^{+}\right\}$et $S\left(\varphi^{-1} N\right)=\left\{a \xi ; a \in \boldsymbol{R}^{+}\right\}$sont équivalents, où $\varphi^{-1} N$ est le noyau de convolution dont la densité par rapport à $N$ est égale $\grave{a} \varphi^{-1}$.

Une mesure de Radon réelle $\eta$ est dite $N_{0}$-harmonique si, pour tout $t \geqq 0, \eta=\eta * \alpha_{t}$, où $\left(\alpha_{t}\right)_{t \geqq 0}$ est le semi-groupe vaguement continu associé $\grave{a}$ $N_{0}$. Dans (b), $N_{0}$ est la plus petite majorante de $N$ (voir [11]).

Démonstration. Soit $\left(\alpha_{t}\right)_{t \geqq 0}$ un semi-groupe vaguement continu vérifiant $\alpha_{t} \neq \varepsilon(t>0)$ tel que $N$ soit conditionnellement sous-médian par rapport à $\left(\alpha_{t}\right)_{t \geqq 0}$ et que, pour $N^{\prime \prime} \in S(N)$ et $t \in \boldsymbol{R}^{+}$quelconques, $N^{\prime \prime} \geqq N^{\prime \prime} * \alpha_{t}$. 
Pour $p>0$ quelconque, on pose $N_{p}=\int_{0}^{\infty} \exp (-p t) \alpha_{t} d t$. Alors, pour $N^{\prime \prime}$ $\in S(N)$ quelconque, $N^{\prime \prime} \geqq p N^{\prime \prime} * N_{p}$.

Supposons d'abord qu'il existe $p_{0}>0$ et $N_{0}^{\prime \prime} \neq 0 \in S(N)$ tels que $N_{0}^{\prime \prime} \neq p_{0} N_{0}^{\prime \prime} * N_{p_{0}}$. Dans ce cas, on montrera que (b) a lieu. D'après la proposition $24, \sum_{n=1}^{\infty}\left(p_{0} N_{p_{0}}\right)^{* n}$ converge vaguement. Alors $N_{0}=\int_{0}^{\infty} \alpha_{t} d t$ existe et $N_{0}=\sum_{n=1}^{\infty} p_{0}^{n-1}\left(N_{p_{0}}\right)^{* n}$. D'après les définitions, $N_{0}$ est un noyau de convolution de Hunt et $N$ est conditionnellement sous-médian par rapport à $N_{0}$. On a évidemment $N \prec N_{0}$. D'après la proposition 10, on a, pour $N^{\prime \prime} \in S(N)$ et $p>0$ quelconques, $N_{p} \prec N^{\prime \prime}$, et donc $N_{0} \prec N^{\prime \prime}$. Par conséquent, $N_{0}$ est la plus petite majorante de $N$. L'égalité (3.11) et l'unicité de $N_{0}$ sont déjà connues (voir la proposition 31 et la remarque 43 dans [11]). On voit que (b) a lieu.

Supposons ensuite que, pour $p>0$ et $N^{\prime \prime} \in S(N)$ quelconques, $N^{\prime \prime}=p N^{\prime \prime} * N_{p}$. Dans ce cas, on montrera que (a) a lieu. On remarque que, pour $p>0$ et $q>0$ quelconques, $\operatorname{supp}\left(N_{p}\right)=\operatorname{supp}\left(N_{q}\right)$ et que $\operatorname{supp}(N) \ni 0$. Montrons que, pour $p>0, \operatorname{supp}(N) \subset \operatorname{supp}\left(N_{p}\right)$. Supposons contrairement que cette inclusion n'a pas lieu. Alors il existe deux fonctions $f \neq 0$ et $g \neq 0 \in C_{K}^{+}$et $y \in X$ tels que supp $(f) \subset\{x \in X ; g(x)>0\}$, $N * f(y)>0$ et que, pour $p>0$ quelconque, $N_{p} * g(y)=0$. Comme $\lim _{p \rightarrow 0} p N * N_{p}$ $=0$ (vaguement), il existe $p_{0}>0$ tel que $N *\left(\varepsilon-p_{0} N_{p_{0}}\right) * f(y)>0$. Comme $\operatorname{supp}\left(N_{p_{0}}\right) \ni 0$, il existe une constante $a>0$ telle que

$$
N *\left(\varepsilon-p_{0} N_{p_{0}}\right) * f \leqq a N_{p_{0}} * g \text { sur } \operatorname{supp}(f) .
$$

De la même manière que dans le théorème $26((\mathrm{~b}) \not \mathrm{a}))$, on a $N *\left(\varepsilon-p_{0} N_{p_{0}}\right) * f$ $\leqq a N_{p_{0}} * g$ sur $X$, d'où une contradiction. Par conséquent, $\operatorname{supp}(N)$ $\subset \operatorname{supp}\left(N_{p}\right)(p>0)$, et donc le sous-groupe fermé engendré par $\operatorname{supp}\left(N_{p}\right)$ est égal à $X$. Remarquons que, pour $N^{\prime \prime} \in S(N)$ quelconque, $N^{\prime \prime}=N^{\prime \prime} * N_{1}$. En utilisant le théorème 2 dans [3], on voit qu'il existe une fonction exponentielle $\varphi(x)>0$ sur $X$ telle que

$$
\varphi(x)=\varphi * N_{1}(x)=\left(\int \frac{1}{\varphi} d N_{1}\right) \varphi(x) \text { sur } X,
$$

car $\left\{M\right.$ : noyau de convolution; $\left.M=M * N_{1}\right\}$ est vaguement fermé. Donc $\int \varphi^{-1} d N_{1}=1$. Comme $\varphi^{-1}$ est aussi exponentielle, on voit que $\left(\varphi^{-1} N_{p}\right)_{p>0}$ est aussi une résolvante, et pour $p>0$ quelconque, $p \int \varphi^{-1} d N_{p}=1$. On 
voit facilement que $S\left(\varphi^{-1} N\right)=\left\{\varphi^{-1} N^{\prime \prime} ; N^{\prime \prime} \in S(N)\right\}$. D'après la proposition 10 et (3.13), on a, pour tout $p>0, \varphi \xi \in S\left(N_{p}\right)$. De la même manière que dans le théorème 26 ((b) $\Rightarrow(\mathrm{a}))$, on a $\varphi \xi \in S(N)$, et donc $S\left(\varphi^{-1} N\right) \ni \xi$. Supposons que $S(N) \neq\left\{a \varphi \xi ; \alpha \in R^{+}\right\}$. Comme $\operatorname{supp}(N) \ni 0,0 \in S(N)$. Donc il existe $N^{\prime \prime} \neq 0 \in S\left(\varphi^{-1} N\right)$ tel que, pour tout $a>0, N^{\prime \prime} \neq a \xi$. Alors il existe $f \neq 0 \in C_{K}^{+}$telle que, $\min \left(N^{\prime \prime} * f, 1\right)$ ne soit pas une constante. Comme, pour $p>0$ quelconque,

$$
\min \left(N^{\prime \prime} * f, 1\right) \geqq\left(\min \left(N^{\prime \prime} * f, 1\right)\right) *\left(p \varphi^{-1} N_{p}\right),
$$

on a $\left(\min \left(N^{\prime \prime} * f, 1\right)\right) \xi \in S\left(\varphi^{-1} N\right)$, et donc $\left(\min \left(N^{\prime \prime} * f, 1\right)\right) \varphi \xi \in S(N)$. D'après notre hypothèse, on a, pour $p>0$ quelconque,

$$
\left(\min \left(N^{\prime \prime} * f, 1\right)\right) *\left(p \varphi^{-1} N_{p}\right)=\min \left(N^{\prime \prime} * f, 1\right) .
$$

En utilisant le théorème 1 dans [3], on voit que $\min \left(N^{\prime \prime} * f, 1\right)$ est une constante sur le sous-groupe fermé engendré par $\operatorname{supp}\left(\varphi^{-1} N_{p}\right)$; c'est-à-dire, $\min \left(N^{\prime \prime} * f, 1\right)$ est une constante. Cela est une contradiction. On voit ainsi que (a) a lieu. La démonstration est complète.

Dans le présent théorème, il est très naturel de supposer que le sous-groupe fermé $X^{\prime}$ engendré par supp $(N)$ est égal à $X$, car, en général, il suffit de considérer la restreinte de $N$ sur $X^{\prime}$ au lieu de $N$.

Remarque 31. Soient $N$ et $N^{\prime}$ les mêmes que dans le théorème 30. Supposons qu'il existe un noyau de convolution $\neq 0$ non-proportionel à $N^{\prime}$ dans $S(N)$. Alors le semi-groupe vaguement continu $\left(\alpha_{t}\right)_{t \geqq 0}$ obtenu dans la remarque 28 est uniquement déterminé excepte la multiplication constante (cf. la question 29).

Cela est un résultat immédiat de l'unicité de la plus petite majorante de $N$ (excepté la multiplication constante).

Le théorème 30 donne immédiatement le corollaire suivant:

Corollaire 32. Soient $N, N^{\prime}$ les mêmes que dans le théorème 30 et $\left(\alpha_{t}\right)_{t \geqq 0}$ un semi-groupe vaguement continu obtenu dans la remarque 28. Alors il existe une fonction exponentielle $\varphi(x)>0$ sur $X$ telle que $\left(\varphi^{-1} \alpha_{t}\right)_{t \geqq 0}$ soit un semi-groupe vaguement continu et markovien $^{(6)}$, ou bien $N_{0}=\int_{0}^{\infty} \alpha_{t} d t$ a un sens.

(6) On dit qu'un semi-groupe vaguement continu $\left(\alpha_{t}\right)_{t \geqq 0}$ est markovien si, pour tout $t>0, \int d \alpha_{t}=1$. 
Démonstration. On peut supposer que $N \neq 0$. Soit $X^{\prime}$ le sous-groupe fermé engendré par supp $(N)$. Alors, pour $t \in R^{+}$quelconque, $\operatorname{supp}\left(\alpha_{t}\right) \subset X^{\prime}$. Supposons que $\int_{0}^{\infty} \alpha_{t} d t$ n'a pas un sens. Comme toute la fonction exponentielle $>0$ sur $X^{\prime}$ peut être prolongée en une fonction exponentielle $>0$ sur $X$, le théorème 30 donne qu'il existe une fonction exponentielle $\varphi(x)>0$ sur $X$ tel que $S(N) \supset\left\{a \varphi \xi ; a \in R^{+}\right\}$. Comme $\varphi \geqq \varphi * \alpha_{t},\left(\varphi^{-1} \alpha_{t}\right)_{t \geq 0}$ est un semi-groupe vaguement continu et sous-markovien. Posons, pour $p>0$ quelconque, $N_{p}=\int_{0}^{\infty} \exp (-p t) \alpha_{t} d t$; alors $\varphi=\varphi *\left(p N_{p}\right)$, et donc, pour $t \in \boldsymbol{R}^{+}$quelconque, $\int \varphi^{-1} d \alpha_{t}=1$, d'où le corollaire 32 .

On aura aussi le corollaire suivant:

CoRollaIRe 33. Soit $N$ un noyau de convolution régulier par rapport $\grave{a} \xi$. Supposons que le sous-groupe fermé engendré par $\operatorname{supp}(N)$ est égal $\grave{a} X$. Si $N$ vérifie le principe classique du maximum, alors on a (a) ou bien (b).

(a) $S(N)=\left\{a \xi ; a \in \boldsymbol{R}^{+}\right\}$.

(b) Il existe un noyau de convolution de Hunt sous-markovien $N_{0}^{(7)}$ tel que $N$ soit conditionnellement sous-médian par rapport à $N_{0}$ et que

$$
S(N)=\overline{\left\{N_{0} * \lambda+a \xi ; \lambda \in D^{+}\left(N_{0}\right), a \in R^{+}\right\},}
$$

où l'adhèrence est au sens de la topologie vague.

Démonstration. Soit $\left(\alpha_{t}\right)_{t \geqq 0}$ le semi-groupe vaguement continu obtenu dans la remarque 28. Comme $S(N) \ni \xi,\left(\alpha_{t}\right)_{t \geqq 0}$ est sous-markovien. Supposons que (a) n'a pas lieu. Alors, d'après le théorème $30, N_{0}=\int_{0}^{\infty} \alpha_{t} d t$ a un sens. Soit $N^{\prime} \in S(N)$ quelconque. Pour $f \neq 0 \in C_{K}^{+}$et $a \in R^{+}$quelconque, $\quad\left(\min \left(N^{\prime} * f, a\right)\right) \xi \in S(N)$. D'après le théorème 30 , il existe $\lambda_{f, a} \in D^{+}\left(N_{0}\right)$ et une mesure de Radon positive et $N_{0}$-harmonique $\eta_{f, a}$ telles que

$$
\left(\min \left(N^{\prime} * f, a\right)\right) \xi=N_{0} * \lambda_{f, a}+\eta_{f, a} .
$$

On remarque ici que, pour tout $t \geqq 0, \int d \alpha_{t}=1$ ou bien que, pour tout

(7) On dit qu'un noyau de convolution de Hunt est sous-markovien si le semigroupe associé est sous-markovien. 
$t>0, \int d \alpha_{t}<1$. Si, pour $t>0, \int d \alpha_{t}<1$, alors $\eta_{f, a}=0$. Supposons $\int d \alpha_{t}=1$. D'après le théorème 1 dans [3], tout le point du sous-groupe fermé engendré par supp $\left(N_{0}\right)$ est une période de $\eta_{f, a}$, car $\eta_{f, a}$ est borneé. Comme le sous-groupe fermé engendré par $\operatorname{supp}\left(N_{0}\right)$ est égal à $X, \eta_{f, a}$ est proportionnelle à $\xi$. Ceux-ci donnent que (b) a lieu. La démonstration est ainsi complète.

En généralisant partiellement le théorème 2 dans [10], on obtiendra le théorème suivant:

THÉORÈme 34. Soit $N$ un noyau de convolution et supposons qu'il existe $N^{\prime} \neq 0 \in S(N)$ tel que $N$ soit régulier par rapport à $N^{\prime}$. Alors il existe deux semi-groupes vaguement continus $\left(\alpha_{t}\right)_{t \geqq 0}$ et $\left(\beta_{t}\right)_{t \geqq 0}$ tels que, pour $t \in \boldsymbol{R}^{+}$quelconque, $N^{\prime} \geqq N^{\prime} * \alpha_{t}$ et $N^{\prime} \geqq N^{\prime} * \beta_{t}$, pour $t>0$ quelconque, $\alpha_{t} \neq \varepsilon$ et que, pour $p>0$ et $q>0$ quelconques,

$$
\left(N *\left(\varepsilon-p N_{p}\right)+q N_{p}\right) * N_{q}^{\prime}=N_{p},
$$

où $N_{p}=\int_{0}^{\infty} \exp (-p t) \alpha_{t} d t$ et où $N_{q}^{\prime}=\int_{0}^{\infty} \exp (-q t) \beta_{t} d t$.

Démonstration. On peut supposer que $N \neq 0$, car si $N=0$, alors, en posant $\alpha_{t}=\exp (-t) \varepsilon$ et $\beta_{t}=\varepsilon,\left(\alpha_{t}\right)_{t \geqq 0}$ et $\left(\beta_{t}\right)_{t \geqq 0}$ sont les deux semigroupes vaguement continus demandés. Soit a une constante $>0$ quelconque. Soit $\mu_{a}^{\prime \prime}$ une mesure balayée transitivement de $\varepsilon$ sur $C\{0\}$ par rapport à $S(N+a \varepsilon)$ vérifiant $\mu_{a}^{\prime \prime}(\{0\})=0$. On note $\left(\alpha_{a, t}\right)_{t \geqq 0}$ et $\left(\beta_{a, t}\right)_{t \geqq 0}$ les deux semi-groupes vaguement continus définis par $\mu_{a}^{\prime \prime}$ de la même manière. que dans le théorème 26. Soit $c_{a}$ une constante $>0$ définie dans la démonstration $\mathrm{du}$ théorème 26. Posons, pour $p>0$ quelconques, $N_{p, a}=\int_{0}^{\infty} \exp (-p t) \alpha_{a, c_{a} t} d t$ et $N_{p, a}^{\prime}=\int_{0}^{\infty} \exp (-p t) \beta_{a, c_{a} t} d t$. Alors, pour $p>0$ quelconque, $N^{\prime} \geqq N^{\prime} *\left(p N_{p, a}\right)$ et $N^{\prime} \geqq N^{\prime} *\left(p N_{p, a}^{\prime}\right)$, d'après la proposition 16. Pour $p>0$ et $q>0$ quelconques, on a

$$
c_{a}\left(\varepsilon-\mu_{a}^{\prime \prime}\right) * N_{p, a}=\varepsilon-p N_{p, a} \text { et } c_{a}(N+a \varepsilon) *\left(\varepsilon-\mu_{a}^{\prime \prime}\right) * N_{q, a}^{\prime}=\varepsilon-q N_{q, a}^{\prime},
$$

et donc

$$
\left((N+a \varepsilon) *\left(\varepsilon-p N_{p, a}\right)+q N_{p, a}\right) * N_{q, a}^{\prime}=N_{p, a} .
$$

De la même manière que dans le théorème 26 , on peut supposer que 
$\left(N_{1^{\prime} a}\right)_{a>0}$ converge vaguement vers un noyau de convolution $N_{1}$ sur $X$ lorsque $a \rightarrow 0$.

Supposons d'abord que $N^{\prime}=N^{\prime} * N_{1}$. De la même manière que dans le théorème 26, on voit que, pour $p>0$ quelconque, $N_{p}=\lim _{a \rightarrow 0} N_{p, a} \neq 0$ (vaguement) existe, $\left(N_{p}\right)_{p>0}$ est une résolvante et que $N^{\prime}=N^{\prime} *\left(p N_{p}\right)$ $=\lim _{a \rightarrow 0} N^{\prime} *\left(p N_{p, a}\right) \quad$ (vaguement). Soit $\left(\alpha_{t}\right)_{t \geqq 0}$ le semi-groupe vaguement continu associé à $\left(N_{p}\right)_{p>0}$. Alors on voit aussi que, pour $t>0$ quelconque, $\alpha_{t} \neq \varepsilon$ et que $N$ soit conditionnellement sous-médian par rapport à $\left(\alpha_{t}\right)_{t \geqq 0}$. Pour $q>0$ quelconque, on désigne par $N_{q}^{\prime}$ un point vaguement adhérent de $\left(N_{q, a}^{\prime}\right)_{a>0}$ lorsque $a \rightarrow 0$ et on choisit une famille filtrante à gauche $\left(a_{\alpha}\right)_{\alpha \in A}$ des nombres $>0$ telle que $\lim _{\alpha} a_{\alpha}=0$ et que $N_{q}^{\prime}=\lim _{\alpha} N_{q, a_{\alpha}}^{\prime}$ (vaguement). De la même manière que dans la proposition 23 , on voit que, pour $p>0$ et $q>0$ quelconques,

$$
\lim _{\alpha} N_{p, a_{\alpha}} * N_{q, a_{\alpha}}^{\prime}=N_{p} * N_{q}^{\prime} \quad \text { (vaguement). }
$$

En utilisant le lemme 17 et (3.20), on a

$$
\left(N *\left(\varepsilon-p N_{p}\right)+q N_{p}\right) * N_{q}^{\prime}=N_{p} \quad(p>0, q>0) .
$$

On a, en même temps, $N_{q}^{\prime} \neq 0$. Pour $p>0$ et $q>0$ quelconques, on a

$$
\begin{aligned}
N_{1} * N_{p}^{\prime} & =\left(N *\left(\varepsilon-N_{1}\right)+q N_{1}\right) * N_{q}^{\prime} * N_{p}^{\prime} \\
& =\left(N *\left(\varepsilon-N_{1}\right)+p N_{1}\right) * N_{q}^{\prime} * N_{p}^{\prime}+(q-p) N_{1} * N_{q}^{\prime} * N_{p}^{\prime} \\
& =N_{1} *\left(N_{q}^{\prime}+(q-p) N_{p}^{\prime} * N_{q}^{\prime}\right),
\end{aligned}
$$

et donc l'injectivité de $N_{1}$ donne que $N_{p}^{\prime}-N_{q}^{\prime}=(q-p) N_{p}^{\prime} * N_{q}^{\prime}$. Ceci donne que, pour $p>0$ quelconque, $N_{p}^{\prime}=\lim _{a \rightarrow 0} N_{p, a}^{\prime}$ (vaguement) existe et que $\left(N_{p}^{\prime}\right)_{p>0}$ est une résolvante. Soit $\left(\beta_{t}\right)_{t \geqq 0}$ le semi-groupe vaguement continu associé à $\left(N_{p}^{\prime}\right)_{p>0}$. Comme $N^{\prime} \geqq p N^{\prime} * N_{p}^{\prime}(p>0)$, on a, pour $t \in R^{+}$ quelconque, $N^{\prime} \geqq N^{\prime} * \beta_{t}$. On voit ainsi que $\left(\alpha_{t}\right)_{t \geqq 0}$ et $\left(\beta_{t}\right)_{t \geqq 0}$ sont deux semi-groupes vaguement continus demandés.

Supposons que $N^{\prime} \neq N^{\prime} * N_{1}$. En regardant la proposition 24 et le théorème 26, on voit qu'il existe un noyau de convolution de Hunt $N_{0}=\int_{0}^{\infty} \alpha_{t} d t$, et un seul tel que $N_{1}=\int_{0}^{\infty} \exp (-t) \alpha_{t} d t$ et que $N$ soit conditionnellement sous-médian par rapport à $N_{0}$. On a aussi $N \prec N_{0}$ (voir (3.3) et (3.4)), et donc $N=O\left(N_{0}\right)$. D'après le théorème 2 dans [10] (ou bien le théorème 32 dans [11]), il existe un noyau de convolution de Hunt 
$N^{\prime}=\int_{0}^{\infty} \beta_{t} d t$, et un seul tel que

$$
N * N^{\prime}=N_{0} .
$$

Soient $\left(N_{p}\right)_{p>0}$ la résolvante associée à $N_{0}$ et $\left(N_{p}^{\prime}\right)_{p>0}$ la résolvante associée à $N^{\prime}$. En utilisant l'équation résolvante, on voit que (3.24) donne (3.18), d'où $\left(\alpha_{t}\right)_{t \geqq 0}$ et $\left(\beta_{t}\right)_{t \geqq 0}$ sont deux semi-groupes vaguement continus demandés. La démonstration est ainsi complète.

CorollaIre 35. Soient $N, N^{\prime},\left(\alpha_{t}\right)_{t \geqq 0}$ et $\left(\beta_{t}\right)_{t \geqq 0}$ les mêmes que dans le théorème 34. Alors il existe la mesure singulière $\beta$ associée à $\left(\beta_{t}\right)_{t \geqq 0}{ }^{\left({ }^{(8)}\right.}$ et, pour $f \in C_{K}^{+}(C\{0\}) \cap \mathscr{D}^{+}\left(\lim _{t \rightarrow 0} \frac{1}{t}\left(N * \alpha_{t}-N\right)\right)$ quelconque,

$$
\lim _{t \rightarrow 0} \frac{1}{t}\left(N * \alpha_{t}-N\right) * f(0)=\int \check{f} d \beta .
$$

Démonstration. Comme, pour $t \in \boldsymbol{R}^{+}$quelconque, $N^{\prime} \geqq N^{\prime} * \beta_{t}$, la proposition 15 et (2.40) donnent qu'il existe la mesure singulière $\beta$ associée à $\left(\beta_{t}\right)_{t \geq 0}$. Soit $\alpha^{\prime}$ la mesure de Radon positive en dehors de l'origine telle que, pour $f \in C_{K}^{+}(C\{0\}) \cap \mathscr{D}^{+}\left(\lim _{t \rightarrow 0} \frac{1}{t}\left(N * \alpha_{t}-N\right)\right)$ quelconque,

$$
\lim _{t \rightarrow 0} \frac{1}{t}\left(N * \alpha_{t}-N\right) * f(0)=\int \check{f} d \alpha^{\prime} .
$$

Soient $N_{p}$ et $N_{p}^{\prime}$ les mêmes que dans le théorème 34. Soit $v \in \mathscr{V}$ quelconque. Soient $\mu_{C v, p}^{(1)}$ et $\mu_{C v, p}^{(2)}$ la mesure balayée de $\varepsilon$ sur $C v$ relativement à $N_{p}$ et celle relativement à $N_{p}^{\prime}$, respectivement. Posons $\mu_{C v}^{(j)}=\lim _{p \rightarrow 0} \mu_{C v, p}^{(j)}$ $(j=1,2), \quad \eta_{v}=\lim _{p \rightarrow 0}\left(N_{p}-N_{p} * \mu_{C v, p}^{(1)}\right) \quad$ et $\quad \eta_{v}^{\prime}=\lim _{p \rightarrow 0}\left(N_{p}^{\prime}-N_{p}^{\prime} * \mu_{C v, p}^{(2)}\right) \quad$ (voir la proposition 15). On a $\eta_{v} \neq 0$ et $\eta_{v}^{\prime} \neq 0$. D'après (3.18), on $\mathrm{a}$, pour $p>0$ et $q>0$ quelconques,

$$
\begin{aligned}
& \left(N *\left(\varepsilon-p N_{p}\right) *\left(\varepsilon-\mu_{C v, p}^{(1)}\right)+q\left(N_{p}-N_{p} * \mu_{C v, p}^{(1)}\right)\right) *\left(N_{q}-N_{q} * \mu_{C v, q}^{(2)}\right) \\
& \quad=\left(N_{p}-N_{p} * \mu_{C v, p}^{(1)}\right) *\left(\varepsilon-\mu_{C v, q}^{(2)}\right) .
\end{aligned}
$$

On a $\lim _{p \rightarrow 0} p N_{p}=0$ (vaguement) et $N^{\prime} \geqq N^{\prime} * \mu_{C v, p}^{(j)}(p>0)$. En faisant $p \downarrow 0$ et ensuite $q \downarrow 0$ dans (3.26), on a, d'après le lemme 17,

$$
N *\left(\varepsilon-\mu_{C v}^{(1)}\right) * \eta_{v}^{\prime}=\eta_{v} *\left(\varepsilon-\mu_{C v}^{(2)}\right) .
$$

(8) Lorsque $\beta_{t} / t$ converge vaguement dans $C\{0\}$ lorsque $t \downarrow 0$, sa limite en dehors de $\{0\}$ s'appelle la mesure singulière associée à $\left(\beta_{t}\right)_{t \geqq 0}$. 
Ceci donne que

$$
\lim _{t \rightarrow 0} \frac{1}{t}\left(N * \alpha_{t}-N\right) *\left(\eta_{v} * \eta_{v}^{\prime}\right)=\lim _{t \rightarrow 0} \frac{1}{t}\left(\beta_{t}-\varepsilon\right) *\left(\eta_{v} * \eta_{v}^{\prime}\right)
$$

(vaguement) (voir (2.40) et (2.41)). Donc, pour $f \in C_{K}^{+}(C\{0\})$ vérifiant $\operatorname{supp}\left(f * \eta_{v} * \eta_{v}^{\prime}\right) \subset C\{0\}$ quelconque,

$$
\int \widetilde{f * \eta_{v}} * \eta_{v}^{\prime} d \beta=\int \widetilde{f * \eta_{v}} * \eta_{v}^{\prime} d \sigma^{\prime}
$$

Comme $v$ est quelconque, $\beta=\alpha^{\prime}$. La démonstration est ainsi complète.

Soit $\hat{X}$ le groupe dual de $X$. On dit qu'une fonction complexe et continue $\psi$ sur $\hat{X}$ est définie-négative si $\psi(\hat{0}) \geqq 0$ (0 est l'origine de $\hat{X}$ ) et si, pour un entier $n \geqq 1,\left(\hat{x}_{j}\right)_{j=1}^{n} \subset \hat{X}$ et $\left(c_{j}\right)_{j=1}^{n}$ de nombres complexes vérifiant $\sum_{j=1}^{n} c_{j}=0$ quelconques,

$$
\sum_{k=1}^{n} \sum_{j=1}^{n} \psi\left(\hat{x}_{j}-\hat{x}_{k}\right) c_{j} \bar{c}_{k} \leqq 0
$$

(cf., par exemple, [1] et [6]). Si $\psi$ est à valeurs réelles, alors $\psi \geqq 0$. On connaît bien que, pour un semi-groupe vaguement continu et sousmarkovien $\left(\alpha_{t}\right)_{t \geqq 0}$ sur $X$ (resp. une fonction définie-négative $\psi$ sur $\hat{X}$ ), il existe une fonction définie-négative $\psi$ sur $\hat{X}$ (resp. un semi-groupe vaguement continu et sous-markovien $\left(\alpha_{t}\right)_{t \geqq 0}$ sur $X$ ), et une seule telle que,

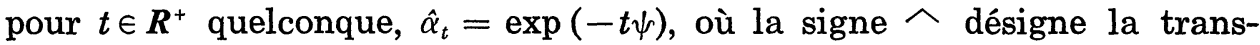
formation de Fourier (cf., par exemple, [6]). Dans ce cas, $\psi\left(\operatorname{resp} .\left(\alpha_{t}\right)_{t \geqq 0}\right)$ s'appelle la fonction définie-négative associée à $\left(\alpha_{t}\right)_{t \geqq 0}$ (resp. le semi-groupe vaguement continu associé à $\psi$ ).

Comme une application du théorème 34 , on montrera complètement le théorème principal dans [7].

THÉORÈme 36. Soit $N$ un noyau de convolution symétrique (par rapport à l'origine) et régulier par rapport à $\xi$. Alors les deux énoncés suivants sont équivalents:

(a) $N$ vérifie le principe classique du maximum.

(b) Il existe deux fonctions définie-négatives $\psi_{1}$ et $\psi_{2}$ à valeurs réelles telles que $\frac{\psi_{2}}{\psi_{1}}$ soit localement sommable et que 


$$
\hat{N}=\frac{\psi_{2}}{\psi_{1}} \hat{\xi}
$$

où $\hat{\xi}$ est la mesure de Haar sur $\hat{X}$ associée $\grave{a} \xi$.

Démonstration. (a) $\Rightarrow(\mathrm{b})$ : D'après le théorème 34 , il existe deux semi-groupes vaguement continus et sous-markoviens $\left(\alpha_{t}\right)_{t \geqq 0}$ et $\left(\beta_{t}\right)_{t \geqq 0}$ tels que, en posant $N_{p}=\int_{0}^{\infty} \exp (-p t) \alpha_{t} d t(p>0)$ et $N_{q}^{\prime}=\int_{0}^{\infty} \exp (-q t) \beta_{t} d t$ $(q>0)$, (3.18) ait lieu. On peut supposer que, pour $a>0$ quelconque, une mesure balayée transitivement $\mu_{a}^{\prime \prime}$ de $\varepsilon$ sur $C\{0\}$ relativement à $(N+a \varepsilon, \xi)$ vérifiant $\mu_{a}^{\prime \prime}(\{0\})=0$ est symétrique par rapport à l'origine. On peut supposer donc que, pour $t \in \boldsymbol{R}^{+}$quelconque, $\alpha_{t}$ et $\beta_{t}$ sont symétrique par rapport à l'origine. Soient $\psi_{1}$ et $\psi_{2}$ la fonction définienégative associée à $\left(\alpha_{t}\right)_{t \geqq 0}$ et celle associée à $\left(\beta_{t}\right)_{t \geqq 0}$, respectivement. Alors $\psi_{j}(j=1,2)$ est à valeurs réelles, et donc $\psi_{j} \geqq 0$. Pour $v \in \mathscr{V}$ quelconque, on désigne par $\mu_{C v}^{\prime}$ la mesure balayée de $\varepsilon$ sur $C v$ relativement à $\left(N_{p}\right)_{p>0}$. Soit $\eta_{v}$ la mesure de Radon positive portée par $v$ telle que, pour $p>0$ quelconque, (2.22) ait lieu; alors $\eta_{v} \neq 0$. Montrons que la variation totale $\left|N * \eta_{v} *\left(\varepsilon-p N_{p}\right)\right|$ de $N * \eta_{v} *\left(\varepsilon-p N_{p}\right)$ est de masse totale finie. D'après (2.22), on a

$$
N * \eta_{v} *\left(\varepsilon-p N_{p}\right)=N * N_{p} *\left(\varepsilon-\mu_{C v}^{\prime}\right),
$$

et donc, (3.18) donne que, pour $q>0$ quelconque,

$$
\left(N_{p} *\left(N-N * \mu_{C v}^{\prime}\right)+q N_{p} * \eta_{v}\right) * N_{q}^{\prime}=N_{p} * \eta_{v} .
$$

D'après la proposition 19, supp $\left(\left(N-N * \mu_{C v}^{\prime}\right)^{+}\right) \subset v$. Comme $\int d N_{p} \leqq \frac{1}{p}$, $\left(N_{p} *\left(N-N * \mu_{C v}^{\prime}\right)+p N_{p} * \eta_{v}\right)^{+}$est de masse totale finie. Comme $N_{q}^{\prime} \sqsubset \xi$, on a

$$
\int d\left(N_{p} *\left(N-N * \mu_{C v}^{\prime}\right)+q N_{p} * \eta_{v}\right) \geqq 0,
$$

et donc $\left|N-N * \mu_{C v}^{\prime}\right|$ est de masse totale finie et $\int d\left(N-N * \mu_{C v}^{\prime}\right)$ $+q \int d \eta_{v} \geqq 0 . \quad$ En faisant $q \downarrow 0$, on arrive à $\int d\left(N-N * \mu_{C v}^{\prime}\right) \geqq 0$. En utilisant (3.32), on voit que $\left|N * \eta_{v} *\left(\varepsilon-p N_{p}\right)\right|$ est de masse totale finie et que $\int d N * \eta_{v} *\left(\varepsilon-p N_{p}\right) \geqq 0$. Posons $\sigma_{v}=\frac{\eta_{v}}{\int d \eta_{v}} ;$ alors $\left|N * \sigma_{v} * \check{\sigma}_{v} *\left(\varepsilon-p N_{p}\right)\right|$ 
est aussi de masse totale finie. D'après (3.18), on a

$$
\widehat{N * \sigma_{v} * \check{\sigma}_{v} *\left(\varepsilon-p N_{p}\right)}=\frac{\psi_{2}}{p+\psi_{1}}\left|\hat{\sigma}_{v}\right|^{2} .
$$

Comme $\lim _{p \rightarrow 0} N *\left(p N_{p}\right)=0$ (vaguement), d'après $\alpha_{t} \neq \varepsilon(t>0)$, et comme $v$ est quelconque, on voit que $N$ est de type positif (au sens des mesures). D'après le théorème de Bochner, $\hat{N}$ a un sens et est une mesure de Radon positive dans $\hat{X}$. Alors, pour $f \in C_{K}$ quelconque,

$$
\int|\hat{f}|^{2} d \hat{N}=N * f * \check{f}(0)=\lim _{v \downarrow\{0\}} \lim _{p \downarrow 0} \int|\hat{f}|^{2}\left|\hat{\sigma}_{v}\right|^{2} \frac{\psi_{2}}{p+\psi_{1}} d \hat{\xi}
$$

Donc $\frac{\psi_{2}}{\psi_{1}}$ est définie $\hat{\xi}$-p.p., localement $\hat{\xi}$-sommable et l'on a

$$
\int|\hat{f}|^{2} d \hat{N}=\int|\hat{f}|^{2} \frac{\psi_{2}}{\psi_{1}} d \hat{\xi}
$$

On a encore, pour $f, g \in C_{K}$ quelconques,

$$
\int \hat{f} \overline{\hat{g}} d \hat{N}=\int \hat{f} \overline{\hat{g}} \frac{\psi_{2}}{\psi_{1}} d \hat{\xi}
$$

Ceci donne immédiatement que $\hat{N}=\frac{\psi_{2}}{\psi_{1}} \hat{\xi}$, d'où (a) $\Rightarrow$ (b).

(b) $\Rightarrow(a)$ : Soient $\left(\alpha_{t}\right)_{t \geqq 0}$ et $\left(\beta_{t}\right)_{t \geqq 0}$ le semi-groupe vaguement continu associé à $\psi_{1}$ et celui associé à $\psi_{2}$, respectivement. Comme $\psi_{1} \neq 0$, on a, pour $t>0$ quelconque, $\alpha_{t} \neq \varepsilon$. Posons, pour $p>0$ quelconque, $N_{p}=\int_{0}^{\infty} \exp (-p t) \alpha_{t} d t$ et $N_{p}^{\prime}=\int_{0}^{\infty} \exp (-p t) \beta_{t} d t$; alors $p \int d N_{p} \leqq 1, p \int d N_{p}^{\prime}$ $\leqq 1$ et $\lim _{p \rightarrow 0} p N_{p}=0$ (vaguement). Comme $f \in C_{K}$ quelconque,

$$
N * f * \check{f}(0)=\int|\hat{f}|^{2} \frac{\psi_{2}}{\psi_{1}} d \hat{\xi}<\infty
$$

$N * f * \check{f}$ s'annule à l'infini. D'après la définition, $N$ s'annule à l'infini. Donc, pour $p>0$ quelconque, $N * N_{p}$ a un sens et $\lim _{p \rightarrow 0} N *\left(p N_{p}\right)=0$ (vaguement). On a, pour $p>0$ et $q>0$ quelconque,

$$
\left(N *\left(\varepsilon-p N_{p}\right)+q N_{p}\right) * N_{q}^{\prime}=N_{p} .
$$

Pour $v \in \mathscr{V}$ quelconque, on désigne par $\mu_{C v, p}^{\prime}$ la mesure balayée de $\varepsilon$ sur 
$C v$ relativement à $N_{p}$. Comme $\lim _{q \rightarrow \infty} q N_{q}^{\prime}=\varepsilon$ (vaguement), on a

$$
\begin{aligned}
N *(\varepsilon & \left.-\mu_{C v, p}^{\prime}\right) *\left(\varepsilon-p N_{p}\right)+p\left(N_{p}-N_{p} * \mu_{C v, p}^{\prime}\right) \\
& =\lim _{q \rightarrow \infty} q\left(N *\left(\varepsilon-\mu_{C v, p}^{\prime}\right) *\left(\varepsilon-p N_{p}\right)+p\left(N_{p}-N_{p} * \mu_{C v, p}^{\prime}\right)\right) * N_{q}^{\prime} \\
& =\lim _{q \rightarrow \infty} q\left(N_{p}-N_{p} * \mu_{C v, p}^{\prime}\right) *\left(\varepsilon-(q-p) N_{q}^{\prime}\right) \leqq 0 \text { dans } C v,
\end{aligned}
$$

d'après (3.40) et l'équation rèsolvante. Soit $\mu_{C v}^{\prime}$ la mesure balayée de $\varepsilon$ sur $C v$ relativement à $\left(N_{p}\right)_{p>0}$. D'après le lemme 17 , on a

$$
N *\left(\varepsilon-\mu_{C v}^{\prime}\right)=\lim _{p \rightarrow 0} N *\left(\varepsilon-\mu_{C v, p}^{\prime}\right) *\left(\varepsilon-p N_{p}\right) \leqq 0 \text { dans } C v .
$$

D'après la proposition 19, $N$ est conditionnellement sous-médian par rapport à $\left(\alpha_{t}\right)_{t \geqq 0}$, et donc le théorème 26 donne que $N \prec \xi$, d'où (b) $\Rightarrow$ (a). La démonstration est ainsi complète.

D'après théorème 36 , on verra facilement le corollaire suivant:

Corollaire 37. Soit $N$ un noyau de convolution symétrique et vérifiant le principe classique du maximum. Alors on $a$ :

(a) Si $N$ est régulier par rapport à $\xi$, alors $N$ s'annule à l'infini.

(b) Si $N$ est régulier par rapport à $\xi$, alors $N$ est de type positif.

On remarque que l'inverse de (a) existe toujours (voir la remarque 5). Dans le théorème 36 , la condition de la symétricité de $N$ est inévitable.

\section{§4. Les noyaux de convolution vérifiant le principe classique $\mathrm{du}$ maximum sur $R^{n}$}

Dans ce paragraphe, on supposera toujours que $X=\boldsymbol{R}^{n}$. On dit qu'une distribution $L$ est un laplacien généralisé si, pour $\varphi \in \mathscr{D}$ à valeurs réelles quelconques,

$$
L(\varphi) \leqq 0
$$

dès que $\varphi(0)=\max _{x \in \boldsymbol{R}^{n}} \varphi(x)$, où $\mathscr{D}$ désigne l'espace vectoriel topologique usuel des fonctions infiniment dérivables dans $\boldsymbol{R}^{n}$ à valeurs complexes et à support compact.

On connaît bien qu'à un semi-groupe vaguement continu et sousmarkovien $\left(\alpha_{t}\right)_{t \geqq 0}$ (resp. un laplacien généralisé $L$ ), on associe uniquement un laplacien généralisé $L$ (resp. un semi-groupe vaguement continu et sous-markovien $\left.\left(\alpha_{t}\right)_{t \geqq 0}\right)$ tel que, pour $\varphi \in \mathscr{D}$ quelconque, 


$$
L * \varphi(0)=\lim _{t \rightarrow 0} \frac{1}{t}\left(\alpha_{t}-\varepsilon\right) * \varphi(0)
$$

(voir, par exemple, [6]). Dans ce cas, $\left(\alpha_{t}\right)_{t \geq 0}$ s'appelle le semi-groupe vaguement continu défini par $L$ et $L$ s'appelle l'opérateur infinitésimal de $\left(\alpha_{t}\right)_{t \geq 0}$.

Ceci donne que, pour un laplacien généralisé $L$ (resp. une fonction définie-négative $\psi$ ), il existe une fonction définie-négative $\psi$ (resp. un laplacien généralisé $L$ ), et une seule telle que

$$
\hat{L}=-\psi d x \text {, }
$$

où la mesure de Lebesgue désigne $d x$.

D'après le théorème de Levy-Khinchine, on connaît que, pour un laplacien généralisé $L$, il existe une constante $c \geqq 0$, une famille $\left(b_{j}\right)_{j=1}^{n}$ des constantes réelles, une matrice réelle et semi-définie-positive $\left(a_{j_{k}}\right)_{j, k=1, \ldots, n}$ et une mesure positive $\sigma$ en dehors de l'origine vérifiant $\int|x|^{2} /\left(1+|x|^{2}\right) d \sigma(x)$ $<\infty$ telles que, pour $\varphi \in \mathscr{D}$ quelconque,

$$
\begin{aligned}
L(\varphi)= & -c \varphi(0)+\sum_{j=1}^{n} b_{j} \frac{\partial \varphi}{\partial x_{j}}(0)+\sum_{k=1}^{n} \sum_{j=1}^{n} a_{j k} \frac{\partial^{2} \varphi}{\partial x_{k} \partial x_{j}}(0) \\
& +\int\left(\varphi(x)-\varphi(0)-\sum_{j=1}^{n} \frac{\partial \varphi}{\partial x_{j}}(0) \frac{x_{j}}{1+|x|^{2}}\right) d \sigma(x),
\end{aligned}
$$

où $x=\left(x_{1}, \cdots, x_{n}\right)$ et $|x|=\left(\sum_{j=1}^{n} x_{j}^{2}\right)^{1 / 2}$.

En utilisant le théorème 26 (et aussi le corollaire 27), on obtiendra l'équivalence explicite du principe classique du maximum.

THÉORÈme 38. Soit $N$ un noyau de convolution sur $\boldsymbol{R}^{n}$ régulier par rapport à $d x$. Alors les deux énoncés suivants sont équivalents:

(a) $N$ vérifie le principe classique du maximum.

(b) $N$ est borné et il existe un laplacien généralisé $L \neq 0$ tel que, au sens des distributions,

$$
L * N \geqq 0 \text { en dehors de l'origine . }
$$

Démonstration. (a) $\Rightarrow$ (b): D'après le corollaire 27, il existe un semigroupe vaguement continu et sous-markovien $\left(\alpha_{t}\right)_{t \geq 0}$ vérifiant $\alpha_{t} \neq \varepsilon(t>0)$ tel que $N$ soit conditionnellement sous-médian par rapport à $\left(\alpha_{t}\right)_{t \geq 0}$. Soit $L$ l'opérateur infinitésimal de $\left(\alpha_{t}\right)_{t \geq 0}$. Alors, d'après $\alpha_{t} \neq \varepsilon(t>0), L \neq 0$. 
En regardant (4.4), on voit qu'il existe un laplacien généralisé $L^{\prime}$ à support compact et une mesure positive $\sigma^{\prime}$ dans $\boldsymbol{R}^{n}$ de masse totale finie tels que

$$
L=L^{\prime}+\sigma^{\prime}-\left(\int d \sigma^{\prime}\right) \varepsilon .
$$

Comme $N$ est borné, $L * N$ a un sens. Soit $\left(N_{p}\right)_{p>0}$ la résolvante vérifiant $N_{p}=\int_{0}^{\infty} \exp (-p t) \alpha_{t} d t(p>0)$. Alors, pour $p>0$ quelconque, $L * N_{p}=p N_{p}$ $-\varepsilon$ (au sens des distributions). Pour $v \in \mathscr{V}$ quelconque, on désigne par $\mu_{C v}^{\prime}$ la mesure balayée de $\varepsilon$ sur $C v$ relativement à $\left(N_{p}\right)_{p>0}$ et par $\eta_{v}$ la mesure positive portée par $v$ vérifiant (2.22). Alors

$$
\begin{aligned}
L * N * \eta_{v} *\left(\varepsilon-p N_{p}\right) & =L * N * N_{p} *\left(\varepsilon-\mu_{C v}^{\prime}\right) \\
& =N *\left(p N_{p}-\varepsilon\right) *\left(\varepsilon-\mu_{C v}^{\prime}\right) .
\end{aligned}
$$

D'après (4.6) et le lemme 17, on a, au sens des distributions,

$$
\lim _{p \rightarrow 0} L * N * \eta_{v} *\left(\varepsilon-p N_{p}\right)=L * N * \eta_{v},
$$

et donc, en utilisant encore le lemme 17 et la proposition 19, on a

$$
L * N * \eta_{v}=N *\left(\mu_{C v}^{\prime}-\varepsilon\right) \geqq 0 \text { dans } C v .
$$

Remarquons que $\eta_{v} \neq 0$. Comme $v$ est quelconque, (4.9) donne immédiatement (4.5), d'où (a) $\Rightarrow$ (b).

(b) $\Rightarrow(\mathrm{a})$ : Soit $\left(\alpha_{t}\right)_{t \geqq 0}$ le semi-groupe vaguement continu défini par L. Comme $L \neq 0$, on a, pour $t>0$ quelconque, $\alpha_{t} \neq \varepsilon$. Soit $\varphi \in \mathscr{D}$ vérifiant $0 \leqq \varphi \leqq 1$ et $\varphi=1$ sur $\left\{x \in \boldsymbol{R}^{n} ;|x| \leqq 1\right\}$. Comme $\lim _{t \rightarrow 0} \frac{1}{t}\left(\alpha_{t}-\varepsilon\right)$ $=L$ (au sens des distributions), on a aussi $\lim _{t \rightarrow 0} \varphi \frac{1}{t}\left(\alpha_{t}-\varepsilon\right)=\varphi L$ et $\lim _{t \rightarrow 0}(1-\varphi) \frac{1}{t}\left(\alpha_{t}-\varepsilon\right)=(1-\varphi) L$ (au sens des distributions). Comme, pour $t>0$ quelconque, $(1-\varphi) \frac{1}{t}\left(\alpha_{t}-\varepsilon\right)$ est une mesure positive, $(1-\varphi) L$ est aussi une mesure positive et $(1-\varphi) \frac{1}{t}\left(\alpha_{t}-\varepsilon\right)$ converge vaguement vers $(1-\varphi) L$ lorsque $t \rightarrow 0$. Soit $v \in \mathscr{V}$ quelconque. Soient $\left(N_{p}\right)_{p>0}, \mu_{C v}^{\prime}$ et $\eta_{v}$ les mêmes que ci-dessus. Alors, pour $f \geqq 0 \in \mathscr{D}$ vérifiant $\operatorname{supp}(f) \subset C v$ quelconque, 


$$
\begin{aligned}
0 \leqq & L * N * \eta_{v}(f)=(\varphi L) * N * \eta_{v}(f)+((1-\varphi) L) * N * \eta_{v}(f) \\
\leqq & \lim _{t \rightarrow 0}\left(\varphi \frac{1}{t}\left(\alpha_{t}-\varepsilon\right)\right) * N * \eta_{v} * \check{f}(0) \\
& +\varliminf_{t \rightarrow 0}\left((1-\varphi) \frac{1}{t}\left(\alpha_{t}-\varepsilon\right)\right) * N * \eta_{v} * \check{f}(0) \\
= & \lim _{t \rightarrow 0} \frac{1}{t}\left(\alpha_{t}-\varepsilon\right) * N * \eta_{v} * \check{f}(0)=\int f d N *\left(\mu_{C v}^{\prime}-\varepsilon\right),
\end{aligned}
$$

d'après (2.41). Par conséquent, la proposition 19 donne que $N$ est conditionnellement sous-médian par rapport à $\left(\alpha_{t}\right)_{t \geq 0}$, et donc, d'après le corollaire 27, $N \prec \xi$. La démonstration est ainsi complète.

En généralisant le résultat de K. Kunugui (cf. [14]), G. Choquet a donné une condition suffisante pour le principe classique du maximum (cf. [2]). Le théorème 38 est un résultat définitif.

En regardant la remarque 28 et le théorème 38 , on verra le corollaire suivant:

Corollaire 39. Soit $N$ un noyau de convolution régulier par rapport $\grave{a} d x$ et vérifiant le principe classique du maximum. Alors il existe un laplacien généralisé $L \neq 0$ tel que $L * N \geqq 0$ au sens des distributions en dehors de l'origine et que, pour $N^{\prime} \in S(N)$ quelconque, $L * N^{\prime} \leqq 0$ au sens des distributions dans $\boldsymbol{R}^{n}$.

En effet, d'après la remarque 28, on voit, de la même manière que dans le théorème 38, qu'il existe un laplacien généralisé $L$ tel que $L * N \geqq 0$ au sens des distributions en dehors de l'origine et que, pour $N^{\prime} \in S(N)$ quelconque, $L * N^{\prime} \leqq 0$ au sens des distributions dans $\boldsymbol{R}^{n}$ dès que $N^{\prime}$ est borné. Pour $N^{\prime} \in S(N)$ quelconque, il existe une suite $\left(N_{m}^{\prime}\right)_{m=1}^{\infty}$ des noyaux de convolution bornés $\subset S(N)$ telle que $\lim _{m \rightarrow \infty} N_{m}^{\prime}=N^{\prime}$ (vaguement) (voir la démonstration du corollaire 33). En rappelant (4.6), on voit que, pour $N^{\prime} \in S(N)$ quelconque, $L * N^{\prime}$ a un sens et que $L * N^{\prime} \leqq 0$ au sens des distributions dans $\boldsymbol{R}^{n}$.

\section{BIBLIOGRAPHE}

[1] A. Beurling et J. Deny, Dirichlet spaces, Proc. Nat. Acad. Sci. U.S.A., 45 (1959), 208-215.

[2] G. Choquet, Sur une large classe de noyaux de convolution satisfaisant au principe du maximum, Sém. Théorie du potentiel, 1958/59.

[ 3 ] G. Choquet et J. Deny, Sur l'équation de convolution $\mu=\mu * \sigma$, C. R. Acad. Sci. Paris, 250 (1960), 4260-4262. 
[4] J. Deny, Noyaux de convolution de Hunt et noyaux associé à une famille fondamentale, Ann. Inst. Fourier, 12 (1962), 643-667.

[5] O. Frostman, Potentiel d'équilibre et capacité des ensembles avec quelques applications à la théorie des fonctions (Thèse), Meddel Lunds Univ. Sem., 3 (1935), $1-118$.

[6] C. S. Herz, Analyse harmonique à plusieurs variables, Sém. Math. d'Orsay, $1965 / 66$.

[7] M. Itô, Sur le principe classique du maximum pour les noyaux de convolution symétriques, Nagoya Math. J., 41 (1971), 121-133.

[8] - Sur la famille sous-ordonnée au noyau de convolution de Hunt II, Nagoya Math. J., 53 (1974), 115-126.

[9] - Caractérisation du principe de domination pour les noyaux de convolution non-bornés, Nagoya Math. J., 57 (1975), 167-197.

[10] — Sur les noyaux de convolution conditionnellement sous-médians, Nagoya Math. J., 66 (1977), 53-76.

[11] —- Sur le principe de domination relatif, le balayage et les noyaux condimennellement sous-médians, J. Math. pures et appl., 57 (1978), 423-451.

[12] J.-P. Kahane, Quotients de fonctions définies-négatives, Sém. Bourbaki, 1966/67.

[13] M. Kishi, Positive idempotents on a locally compact abelian group, Kōdai Math. Sem. Rep., 27 (1976), 181-187.

[14] K. Kunugui, Étude sur la théorie du potentiel généralisé, Osaka Math. J., 2 (1950), 63-102.

Départment de Mathématiques

Université de Nagoya 